University of Louisville

ThinkIR: The University of Louisville's Institutional Repository

Faculty Scholarship

$12-2007$

\title{
Spiral disk opacity from occulting galaxy pairs in the Sloan Digital Sky Survey.
}

Benne W. Holwerda

University of Louisville

W. C. Keel

University of Alabama - Tuscaloosa

A. Bolton

Harvard-Smithsonian Center for Astrophysics

Follow this and additional works at: https://ir.library.louisville.edu/faculty

Part of the Astrophysics and Astronomy Commons

\section{Original Publication Information}

Holwerda, B. W., W. C. Keel and A. Bolton."Spiral Disk Opacity from Occulting Galaxy Pairs in the Sloan Digital Sky Survey." 2007. The Astronomical Journal 134(6): 2385-2397.

This Article is brought to you for free and open access by ThinkIR: The University of Louisville's Institutional Repository. It has been accepted for inclusion in Faculty Scholarship by an authorized administrator of ThinkIR: The University of Louisville's Institutional Repository. For more information, please contact thinkir@louisville.edu. 


\title{
SPIRAL DISK OPACITY FROM OCCULTING GALAXY PAIRS IN THE SLOAN DIGITAL SKY SURVEY
}

\author{
B. W. Holwerda, ${ }^{1}$ W. C. Keel, ${ }^{2}$ and A. Bolton ${ }^{3}$ \\ Received 2007 July 19; accepted 2007 August 20
}

\begin{abstract}
A spiral galaxy partially overlapping a more distant elliptical offers a unique opportunity to measure the dust extinction in the foreground spiral. From the Sloan Digital Sky Survey (SDSS) DR4 spectroscopic sample, we selected 83 occulting galaxy pairs and measured disk opacity over the redshift range $z=0.0-0.2$ with the goal of determining the recent evolution of disk dust opacity. The enrichment of the ISM changes over the lifetime of a disk, and it is reasonable to expect the dust extinction properties of spiral disks as a whole to change over their lifetime. When they do, the change will affect our measurements of galaxies over the observable universe. From the SDSS pairs we conclude that spiral disks show evidence of extinction to $\sim 2$ effective radii. However, no evidence for recent evolution of disk opacity is evident, due to the limited redshift range and our inability to distinguish other factors on disk opacity such as the presence of spiral arms and Hubble type. Such effects also mask any relation between surface brightness and optical depth that has been found in nearby galaxies. Hence, we conclude that the SDSS spectral catalog is an excellent way to find occulting pairs and construct a uniform local sample. However, a higher resolution than that of the SDSS images is needed to disentangle the effects of spiral arms and Hubble type from evolution since $z=0.2$.
\end{abstract}

Key words: dust, extinction — galaxies: evolution — galaxies: fundamental parameters — galaxies: ISM — galaxies: spiral — galaxies: structure

\section{INTRODUCTION}

The role of extinction may well change across the nowobservable range of redshifts, as heavy elements are injected into the ISM but the reservoir of gas within a galaxy is progressively cycled through stars. Thus, the history of dust within galaxies is intimately linked to the history of the overall star formation rate (SFR), which has been at least partly specified through observations of several deep fields with the Hubble Space Telescope (HST) and other instruments. Calzetti \& Heckman (1999) incorporated both SFR and abundance constraints to examine the possible histories of galaxy extinction, finding that these constraints allow two classes of solutions. These have markedly different redshift behavior, one with a peak extinction early on $(z \approx 3)$, and the other building up dust more gradually, with a peak extinction as late as $z=1$.

The typical dust mass in distant galaxies is very much a function of the selected sample. Far-infrared-selected samples point to more Arp 220 - like dust prominence (Rowan-Robinson et al. 2005), optical-/UV-selected samples point to disks very similar to the local ones (Sajina et al. 2006), and Ly $\alpha$ galaxies point to lowextinction disks (Nilsson et al. 2007).

The observational situation at large redshifts is still illconstrained, so far based on (1) modeling the spectral energy distributions (SEDs) of galaxies at high $z$ (Rowan-Robinson 2003; Rowan-Robinson et al. 2005; Sajina et al. 2006; Nilsson et al. 2007), (2) measuring differences in the SEDs of lensed quasars in which one image is formed deep within the lens system (e.g., Nadeau et al. 1991; Falco et al. 1999; Motta et al. 2002; Elíasdóttir et al. 2006), (3) correlating the colors of quasars with metal-line absorption systems compared to average QSO SEDs (Hopkins

\footnotetext{
${ }^{1}$ Space Telescope Science Institute, 3700 San Martin Drive, Baltimore, MD 21218,USA; holwerda@stsci.edu.

2 Department of Physics and Astronomy, University of Alabama, Box 870324, Tuscaloosa, AL 35487-0324, USA.

${ }^{3}$ Harvard-Smithsonian Center for Astrophysics, 60 Garden Street, MS-20, Cambridge, MA 02138, USA.
}

et al. 2004), and (4) the extinction fits to SN1a light curves (e.g., Knop et al. 2003; Jha et al. 2007). To date, the SED models have yielded the most results: Rowan-Robinson (2003) find a peak extinction at $z \sim 1$ and lower beyond that - there is no extinction at $z=6-7$ according to Yan et al. (2005). Yet Vijh et al. (2003) find a strong correction for the SFR in the early universe due to dust attenuation by an LMC-type dust. The QSO SEDs match the SMC's extinction law, with most of the extinction in the nucleus itself. Most of the lensing systems are early types, however. Lensing measurements find a wide range in the values of $R_{V}$, and the SN1a measurements find no change in reddening.

The first three methods are very vulnerable to color and surface brightness selection effects, and the SED models are very dependent on the assumed geometry of ISM and stars. Partially overlapping galaxies offer a alternative and more direct way to approach the question of the evolution of dusty ISM in spirals.

\section{THE OCCULTING GALAXY PAIRS TECHNIQUE}

An occulting galaxy pair-a foreground spiral partially covering a background galaxy — can be used up to high redshift to determine the opacity of the spiral. The flux contributions in the overlap region from both galaxies are estimated from the nonoverlap parts (Figs. 1 and 2). From a single image, we obtain the nonoverlapping flux from the foreground spiral $\left(F^{\prime}\right)$, the nonoverlap background flux $\left(B^{\prime}\right)$, and the flux from the overlap region $\left(F+B e^{-\tau}\right)$. We can now estimate the optical depth $\left(\tau^{\prime}\right)$ from these three observables: $e^{-\tau^{\prime}}=\left[\left(F+B e^{-\tau}\right)-F^{\prime}\right] / B^{\prime}$.

As the background galaxy, a partially occulted elliptical is ideal because its light profile is very symmetric: the assumption that the nonocculted part is a good approximation of the occulted part is a reasonable one. This leaves the assumption that the foreground spiral is symmetric as the predominant source of uncertainty. This method has been extensively used in the local universe, which gives us a direct comparison set for more distant measurements.

The occulting galaxy technique was originally proposed by White \& Keel (1992) to probe spiral disk extinction in the local universe, and in the following decade the known nearby 


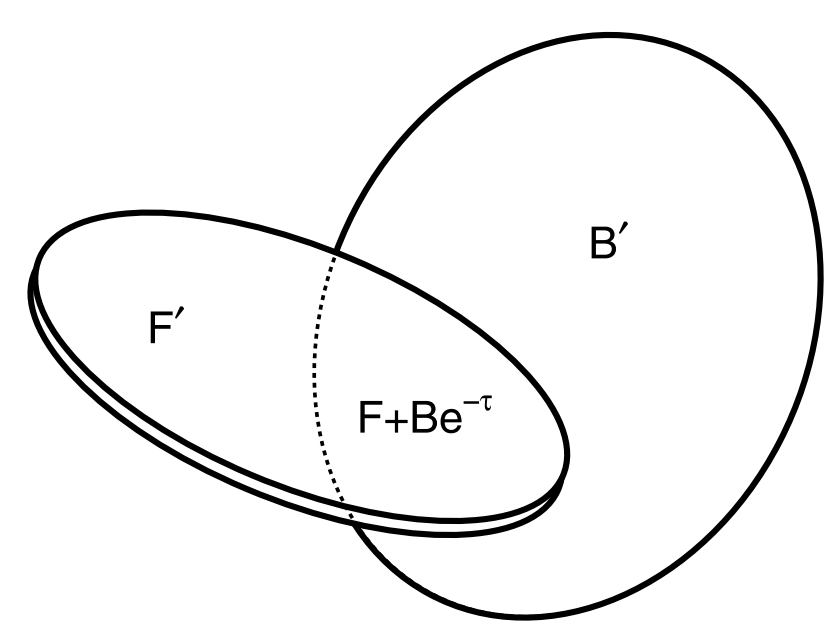

FIG. 1.- Schematic of the ideal occulting pair. The background galaxy is a symmetric elliptical galaxy, and the foreground galaxy a symmetric spiral. In the overlap region, the elliptical is bright enough to provide an extinction signal but the elliptical is not completely hidden behind the spiral, so the contribution of the elliptical to the overlap region can be estimated from the nonocculted part.

overlapping-galaxy pairs were exhausted, using ground-based imaging (Andredakis \& van der Kruit 1992; Berlind et al. 1997; Domingue et al. 1999; White et al. 2000), spectroscopy (Domingue et al. 2000), and HST WFPC2 imaging (Keel \& White 2001a, 2001b; Elmegreen et al. 2001). The ideal pair, described by White \& Keel (1992), is a bright elliptical partially behind a symmetric, face-on spiral. Since only a few pairs were known in the local universe, these studies used a great variety of background galaxies. Their results included radial extinction plots for the extinction across the entire height of the disk. The results follow a gray extinction law when taken over large regions but approach a typical Galactic extinction law for scales smaller than 100 pc (Keel \& White 2001a). Some indication that the cloud sizes are fractal

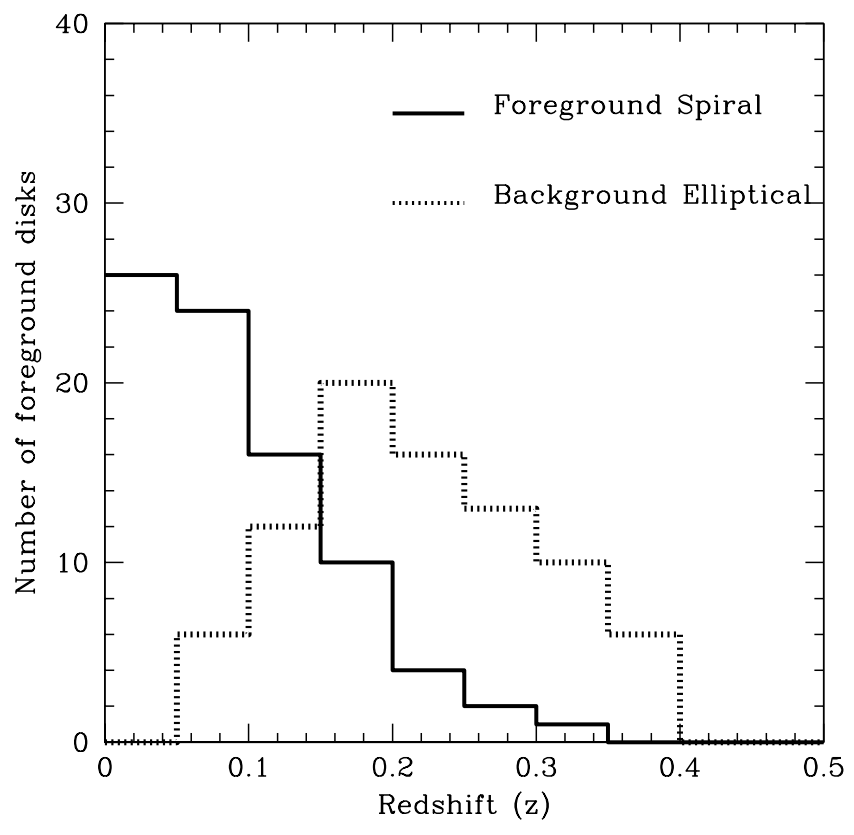

FIG. 3.- Histogram of the redshift distribution of both the background and foreground galaxy. The majority of pairs are nearby $(z<0.1)$. The spectroscopic selection from the SDSS limits the pairs to closer than $z=0.4$.

in nature was found by Keel \& White (2001a). Holwerda et al. (2005a) used counts of distant galaxies seen in HST images of nearby spirals to independently confirm the values for disk extinction from occulting pairs.

From the Sloan Digital Sky Survey (SDSS) DR4 spectroscopic sample we have selected 83 occulting pairs with an elliptical as the background galaxy - the ideal configuration. The foreground spiral galaxies span a range in redshift $(z=0.01-0.3)$. Starting with the spectroscopic identification of both sources and their redshifts represents a significant improvement in reliability and

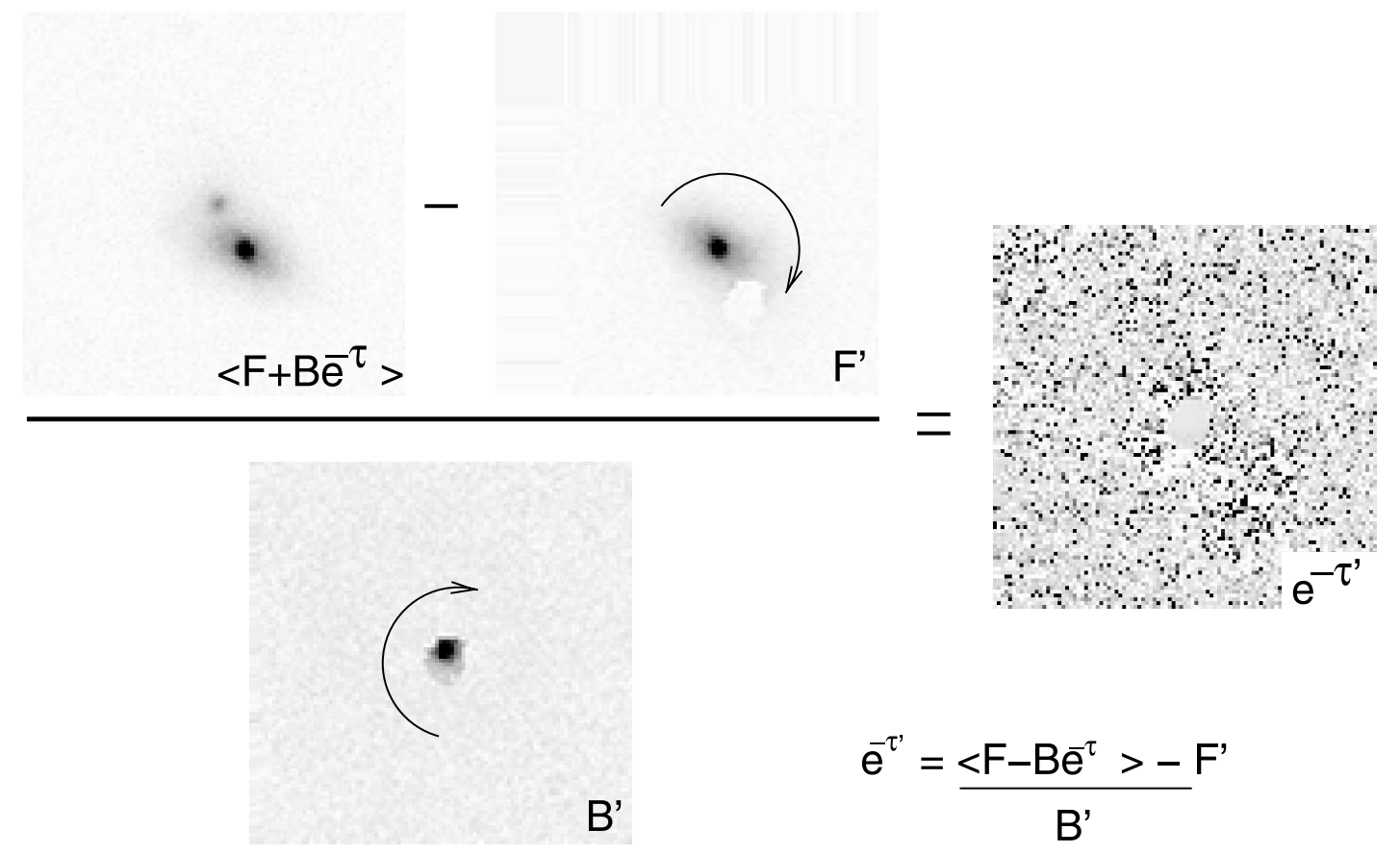

FIG. 2.- Schematic of the automated occulting galaxy method with the pair 1006-52708-624. The extinction map is constructed when the fit parameters (central positions and rotation angles) of both pair members have been determined. The foreground galaxy is rotated and subtracted with the resulting image divided by the flipped background image. The resulting image is the opacity map. In case the area of the background image is perfectly unity, both galaxies are perfectly symmetric and there is no extinction. 
TABLE 1

The SDSS Basic Data of the Occulting Galaxy Pairs

\begin{tabular}{|c|c|c|c|c|c|c|c|c|c|c|c|}
\hline Plate & MJD & Fiber & R.A. & Decl. & $z_{\mathrm{fg}}$ & $z_{\mathrm{bg}}$ & $u$ & $g$ & $r$ & $i$ & $z$ \\
\hline 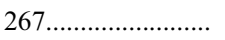 & 51,608 & 499 & 148.311890 & 0.649980 & 0.0355 & 0.0941 & 22.79 & 20.11 & 18.79 & 18.20 & 17.66 \\
\hline $273 \ldots$ & 51,957 & 323 & 156.808510 & 0.680520 & 0.1071 & 0.1781 & 21.23 & 19.62 & 18.54 & 18.01 & 17.63 \\
\hline $275 \ldots \ldots \ldots \ldots \ldots \ldots \ldots \ldots$ & 51,910 & 233 & 161.287840 & 0.075950 & 0.0260 & 0.0944 & 21.22 & 19.10 & 18.07 & 17.54 & 17.24 \\
\hline $281 \ldots \ldots \ldots \ldots \ldots \ldots \ldots$ & 51,614 & 624 & 173.070050 & 0.608420 & 0.0468 & 0.1988 & 22.20 & 20.20 & 19.01 & 18.51 & 18.04 \\
\hline $282 \ldots \ldots \ldots \ldots \ldots \ldots \ldots \ldots$ & 51,658 & 120 & 174.666790 & -0.146470 & 0.0722 & 0.2600 & 23.92 & 21.60 & 19.94 & 19.15 & 18.53 \\
\hline 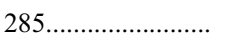 & 51,930 & 15 & 180.269580 & -0.787540 & 0.0213 & 0.1408 & 21.55 & 20.38 & 19.70 & 20.27 & 19.81 \\
\hline 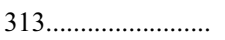 & 51,673 & 546 & 231.835980 & 0.477040 & 0.0413 & 0.3033 & 23.41 & 21.07 & 19.43 & 18.85 & 18.42 \\
\hline 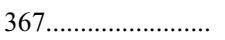 & 51,997 & 222 & 259.541780 & 55.201470 & 0.0836 & 0.1394 & 21.30 & 19.50 & 18.44 & 17.94 & 17.54 \\
\hline 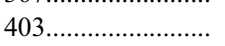 & 51,871 & 586 & 30.145580 & 0.560980 & 0.1378 & 0.1658 & 21.70 & 19.71 & 18.58 & 18.04 & 17.66 \\
\hline 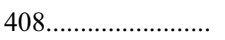 & 51,821 & 336 & 38.992950 & 1.257390 & 0.0682 & 0.1243 & 23.31 & 20.47 & 19.34 & 18.65 & 18.23 \\
\hline 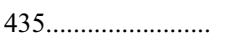 & 51,882 & 118 & 118.882910 & 40.503790 & 0.2313 & 0.2620 & 23.54 & 21.07 & 19.45 & 18.82 & 18.32 \\
\hline 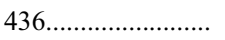 & 51,883 & 638 & 120.747080 & 45.492670 & 0.0389 & 0.3643 & 23.75 & 22.78 & 20.48 & 19.75 & 19.28 \\
\hline $440 \ldots \ldots \ldots \ldots \ldots \ldots \ldots \ldots$ & 51,885 & 370 & 123.106550 & 50.404870 & 0.1756 & 0.2453 & 23.91 & 21.72 & 20.09 & 19.24 & 18.93 \\
\hline $464 \ldots \ldots \ldots \ldots \ldots \ldots \ldots \ldots$ & 51,908 & 211 & 58.942650 & -5.948270 & 0.1230 & 0.2011 & 24.24 & 20.67 & 19.37 & 18.68 & 18.22 \\
\hline 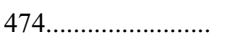 & 52,000 & 548 & 142.017490 & 0.242640 & 0.2061 & 0.2970 & 22.94 & 20.72 & 19.15 & 18.58 & 18.21 \\
\hline 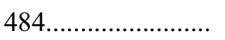 & 51,907 & 616 & 139.814360 & 59.294570 & 0.1582 & 0.2453 & 25.28 & 21.65 & 19.81 & 19.15 & 18.83 \\
\hline 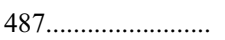 & 51,943 & 305 & 147.228910 & 62.483730 & 0.1245 & 0.2479 & 24.03 & 21.38 & 19.78 & 19.09 & 18.70 \\
\hline $497 \ldots \ldots \ldots \ldots \ldots \ldots \ldots$ & 51,989 & 73 & 208.034660 & 65.742370 & 0.1953 & 0.3958 & 23.67 & 21.82 & 20.06 & 19.23 & 18.63 \\
\hline 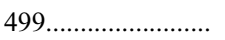 & 51,988 & 152 & 217.024030 & 63.613230 & 0.1145 & 0.2863 & 25.86 & 22.30 & 20.05 & 19.48 & 19.10 \\
\hline 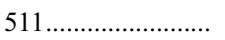 & 52,636 & 313 & 169.186260 & 2.065220 & 0.1292 & 0.2673 & 22.10 & 20.43 & 19.16 & 18.52 & 18.12 \\
\hline $512 \ldots \ldots \ldots \ldots \ldots \ldots \ldots \ldots$ & 51,992 & 302 & 171.158050 & 1.820180 & 0.0492 & 0.2321 & 22.85 & 20.44 & 18.90 & 18.32 & 17.94 \\
\hline 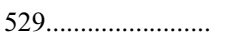 & 52,025 & 408 & 205.440810 & 3.431220 & 0.0232 & 0.1681 & 21.74 & 19.97 & 18.92 & 18.45 & 18.14 \\
\hline 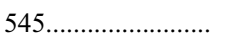 & 52,202 & 504 & 121.639780 & 40.920020 & 0.0764 & 0.3170 & 24.52 & 22.43 & 20.28 & 19.58 & 19.19 \\
\hline 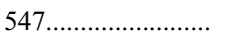 & 52,207 & 167 & 124.385840 & 43.503210 & 0.1415 & 0.3761 & 24.73 & 22.51 & 20.37 & 19.56 & 19.05 \\
\hline $550 \ldots \ldots \ldots \ldots \ldots \ldots \ldots \ldots$ & 51,959 & 624 & 131.782940 & 48.626320 & 0.1743 & 0.1975 & 24.74 & 21.65 & 19.57 & 18.89 & 18.57 \\
\hline 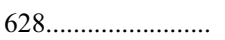 & 52,083 & 262 & 249.181810 & 41.790430 & 0.0284 & 0.1203 & 21.19 & 19.49 & 18.55 & 18.18 & 17.75 \\
\hline 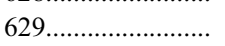 & 52,051 & 26 & 252.894690 & 43.849070 & 0.1152 & 0.3502 & 23.01 & 22.07 & 20.40 & 19.41 & 19.08 \\
\hline 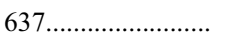 & 52,174 & 334 & 314.058170 & -6.743460 & 0.0878 & 0.1779 & 23.42 & 20.95 & 19.29 & 18.65 & 18.31 \\
\hline $671 \ldots \ldots \ldots \ldots \ldots \ldots$ & 52,206 & 347 & 34.784680 & 0.615480 & 0.0408 & 0.2803 & 22.02 & 21.57 & 20.60 & 20.28 & 19.76 \\
\hline 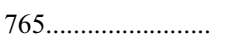 & 52,254 & 179 & 137.082920 & 49.082730 & 0.0355 & 0.2128 & 21.32 & 19.83 & 18.68 & 18.18 & 17.80 \\
\hline $782 \ldots \ldots \ldots \ldots \ldots \ldots \ldots \ldots$ & 52,320 & 306 & 190.878970 & 62.349390 & 0.1452 & 0.2056 & 22.21 & 20.15 & 18.82 & 18.25 & 17.87 \\
\hline 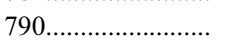 & 52,441 & 527 & 219.458290 & 58.911160 & 0.0317 & 0.1381 & 22.33 & 19.79 & 18.59 & 18.08 & 17.81 \\
\hline 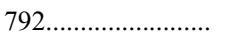 & 52,353 & 432 & 224.462030 & 56.091590 & 0.1423 & 0.2012 & 22.25 & 20.27 & 18.77 & 18.19 & 17.85 \\
\hline $808 \ldots \ldots \ldots \ldots \ldots \ldots \ldots$ & 52,556 & 39 & 46.965700 & -0.311940 & 0.2139 & 0.3731 & 23.21 & 21.72 & 20.34 & 19.66 & 19.27 \\
\hline 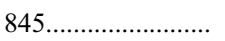 & 52,381 & 270 & 187.675090 & 4.707290 & 0.0700 & 0.3164 & 24.73 & 21.85 & 19.52 & 18.88 & 18.60 \\
\hline 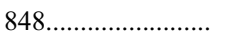 & 52,669 & 602 & 195.053830 & 5.739970 & 0.0490 & 0.2219 & 22.22 & 20.39 & 19.20 & 18.80 & 18.39 \\
\hline $860 \ldots \ldots \ldots \ldots \ldots \ldots \ldots$ & 52,319 & 451 & 121.339030 & 30.762010 & 0.0370 & 0.1607 & 21.94 & 21.15 & 18.59 & 17.94 & 17.59 \\
\hline 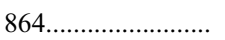 & 52,320 & 91 & 129.972990 & 35.742270 & 0.0439 & 0.2576 & 22.17 & 20.78 & 19.72 & 19.18 & 18.83 \\
\hline 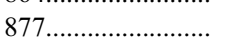 & 52,353 & 458 & 166.000290 & 51.786350 & 0.0370 & 0.0616 & 21.28 & 20.11 & 19.54 & 19.19 & 19.01 \\
\hline $882 \ldots \ldots \ldots \ldots \ldots \ldots \ldots$ & 52,370 & 122 & 180.578770 & 51.309050 & 0.0599 & 0.1828 & 21.51 & 19.41 & 18.26 & 17.76 & 17.45 \\
\hline $883 \ldots \ldots \ldots \ldots \ldots \ldots$ & 52,430 & 366 & 182.350150 & 53.640160 & 0.0492 & 0.1319 & 21.68 & 19.73 & 18.65 & 18.12 & 17.73 \\
\hline $884 \ldots \ldots \ldots \ldots \ldots \ldots \ldots \ldots$ & 52,374 & 242 & 186.059060 & 51.293770 & 0.0413 & 0.1745 & 21.35 & 19.56 & 18.42 & 17.94 & 17.62 \\
\hline $889 \ldots \ldots \ldots \ldots \ldots \ldots \ldots$ & 52,663 & 408 & 116.692660 & 30.657980 & 0.0558 & 0.1612 & 21.44 & 19.77 & 18.57 & 18.02 & 17.63 \\
\hline 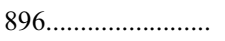 & 52,592 & 463 & 131.060470 & 43.308520 & 0.0275 & 0.1059 & 21.57 & 19.52 & 18.41 & 17.96 & 17.56 \\
\hline 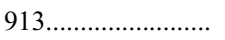 & 52,433 & 151 & 206.813230 & -2.646100 & 0.0843 & 0.1583 & 21.77 & 19.58 & 18.32 & 17.79 & 17.38 \\
\hline 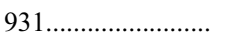 & 52,619 & 84 & 124.763000 & 29.720850 & 0.1835 & 0.2781 & 22.60 & 20.79 & 19.32 & 18.68 & 18.28 \\
\hline 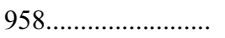 & 52,410 & 194 & 197.246590 & 59.181390 & 0.1523 & 0.3145 & 25.22 & 21.71 & 20.00 & 19.28 & 19.05 \\
\hline 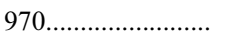 & 52,413 & 408 & 183.318710 & 51.174320 & 0.3046 & 0.3819 & 22.92 & 21.27 & 19.78 & 19.08 & 18.60 \\
\hline 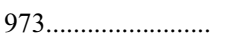 & 52,426 & 519 & 255.808640 & 33.511540 & 0.0631 & 0.0914 & 21.88 & 19.07 & 18.00 & 17.47 & 17.15 \\
\hline 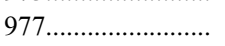 & 52,410 & 539 & 258.531050 & 29.864680 & 0.0824 & 0.1954 & 22.13 & 19.91 & 18.67 & 18.11 & 17.80 \\
\hline 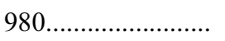 & 52,431 & 300 & 258.937370 & 27.421190 & 0.1253 & 0.2907 & 24.22 & 21.76 & 19.48 & 18.78 & 18.44 \\
\hline $1000 \ldots \ldots \ldots \ldots \ldots \ldots \ldots$ & 52,643 & 337 & 159.372270 & 7.579740 & 0.0851 & 0.3245 & 24.23 & 21.53 & 19.44 & 18.83 & 18.51 \\
\hline $1006 \ldots \ldots \ldots \ldots \ldots \ldots \ldots$ & 52,708 & 624 & 152.436350 & 49.838300 & 0.0524 & 0.1323 & 21.96 & 19.99 & 18.47 & 18.02 & 17.50 \\
\hline $1007 \ldots \ldots \ldots \ldots \ldots \ldots \ldots$ & 52,706 & 519 & 153.131040 & 50.738660 & 0.0463 & 0.1952 & 21.60 & 19.74 & 18.47 & 17.93 & 17.63 \\
\hline $1160 \ldots \ldots \ldots \ldots \ldots \ldots \ldots$ & 52,674 & 468 & 214.447800 & 56.804440 & 0.2381 & 0.2914 & 22.28 & 20.80 & 19.58 & 19.12 & 18.81 \\
\hline 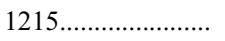 & 52,725 & 288 & 143.281310 & 39.220680 & 0.2708 & 0.3340 & 22.71 & 21.18 & 19.56 & 18.95 & 18.60 \\
\hline 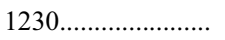 & 52,672 & 639 & 185.753290 & 10.099700 & 0.0536 & 0.1608 & 21.67 & 20.04 & 19.04 & 18.57 & 18.17 \\
\hline $1235 \ldots \ldots \ldots \ldots \ldots \ldots \ldots$ & 52,734 & 164 & 148.326780 & 7.600730 & 0.0956 & 0.3255 & 22.21 & 20.84 & 19.34 & 18.73 & 18.33 \\
\hline $1269 \ldots \ldots \ldots \ldots \ldots \ldots \ldots$ & 52,937 & 485 & 130.654940 & 30.422070 & 0.0272 & 0.1878 & 21.30 & 19.90 & 18.91 & 18.48 & 18.23 \\
\hline $1280 \ldots \ldots \ldots \ldots \ldots \ldots \ldots \ldots$ & 52,738 & 125 & 195.231990 & 48.583740 & 0.0799 & 0.2089 & 21.89 & 20.13 & 18.95 & 18.45 & 18.14 \\
\hline $1282 \ldots \ldots \ldots \ldots \ldots \ldots \ldots$ & 52,759 & 630 & 202.872560 & 49.243360 & 0.1245 & 0.2786 & 21.92 & 20.42 & 19.06 & 18.51 & 18.14 \\
\hline $1310 \ldots \ldots \ldots \ldots \ldots \ldots \ldots$ & 53,033 & 202 & 173.008190 & 56.231840 & 0.1140 & 0.1840 & 21.76 & 19.95 & 18.75 & 18.26 & 17.89 \\
\hline $1317 \ldots \ldots \ldots \ldots \ldots \ldots \ldots$ & 52,765 & 9 & 192.781290 & 56.429170 & 0.0974 & 0.2008 & 21.59 & 19.86 & 18.60 & 18.06 & 17.64 \\
\hline $1321 \ldots \ldots \ldots \ldots \ldots \ldots \ldots$ & 52,764 & 461 & 203.989570 & 56.632780 & 0.1528 & 0.2173 & 22.30 & 20.49 & 19.11 & 18.56 & 18.24 \\
\hline $1324 \ldots \ldots \ldots \ldots \ldots \ldots \ldots$ & 53,088 & 50 & 212.489360 & 53.497750 & 0.0422 & 0.2251 & 22.31 & 20.52 & 19.31 & 18.79 & 18.46 \\
\hline
\end{tabular}


TABLE 1 - Continued

\begin{tabular}{|c|c|c|c|c|c|c|c|c|c|c|c|}
\hline Plate & MJD & Fiber & R.A. & Decl. & $z_{\mathrm{fg}}$ & $z_{\mathrm{bg}}$ & $u$ & $g$ & $r$ & $i$ & $z$ \\
\hline $1325 \ldots \ldots \ldots \ldots$ & 52,762 & 313 & 211.388780 & 54.126820 & 0.1168 & 0.2119 & 22.14 & 20.29 & 18.96 & 18.48 & 18.10 \\
\hline $1327 \ldots$ & 52,781 & 252 & 218.738380 & 51.467310 & 0.0799 & 0.1408 & 21.41 & 19.79 & 18.77 & 18.31 & 17.97 \\
\hline 1332. & 52,781 & 469 & 233.667240 & 46.404020 & 0.2849 & 0.3199 & 22.41 & 20.89 & 19.44 & 18.89 & 18.57 \\
\hline $1363 \ldots \ldots \ldots$ & 53,053 & 104 & 166.400210 & 42.239340 & 0.1759 & 0.2673 & 22.44 & 20.71 & 19.28 & 18.73 & 18.41 \\
\hline $1373 \ldots \ldots \ldots \ldots \ldots \ldots$ & 53,063 & 255 & 191.929650 & 43.917920 & 0.0294 & 0.1317 & 21.48 & 19.63 & 18.61 & 18.20 & 17.87 \\
\hline $1388 \ldots \ldots \ldots \ldots \ldots$ & 53,119 & 618 & 233.838020 & 32.213560 & 0.0370 & 0.2180 & 21.91 & 20.30 & 19.18 & 18.69 & 18.36 \\
\hline 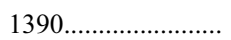 & 53,142 & 385 & 236.455400 & 29.691260 & 0.1313 & 0.2863 & 22.17 & 20.44 & 19.02 & 18.42 & 18.02 \\
\hline $1402 \ldots \ldots \ldots \ldots \ldots \ldots$ & 52,872 & 554 & 236.160490 & 35.176090 & 0.0550 & 0.0790 & 21.02 & 19.26 & 18.35 & 17.90 & 17.60 \\
\hline $1416 \ldots \ldots \ldots \ldots \ldots \ldots \ldots \ldots$ & 52,875 & 490 & 236.353870 & 36.378000 & 0.0653 & 0.1909 & 21.48 & 20.05 & 19.03 & 18.56 & 18.17 \\
\hline 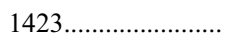 & 53,167 & 528 & 250.832630 & 26.703910 & 0.1975 & 0.3322 & 22.71 & 21.35 & 19.89 & 19.30 & 18.92 \\
\hline 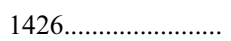 & 52,993 & 385 & 151.191870 & 37.772590 & 0.0232 & 0.0519 & 20.39 & 18.62 & 17.78 & 17.40 & 17.09 \\
\hline $1429 \ldots \ldots \ldots \ldots \ldots \ldots \ldots$ & 52,990 & 336 & 156.275830 & 45.059290 & 0.0742 & 0.1461 & 21.44 & 19.68 & 18.50 & 17.97 & 17.62 \\
\hline 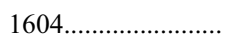 & 53,078 & 459 & 168.059410 & 12.811400 & 0.0771 & 0.1681 & 21.30 & 19.22 & 18.03 & 17.54 & 17.15 \\
\hline $1607 \ldots \ldots \ldots \ldots \ldots \ldots$ & 53,083 & 48 & 174.721650 & 11.288210 & 0.0814 & 0.1513 & 21.59 & 19.93 & 18.80 & 18.42 & 18.00 \\
\hline 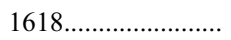 & 53,116 & 285 & 171.000090 & 6.105510 & 0.0370 & 0.1741 & 21.50 & 19.68 & 18.56 & 18.11 & 17.74 \\
\hline $1620 \ldots \ldots \ldots \ldots$ & 53,137 & 175 & 175.693770 & 7.235100 & 0.0653 & 0.1011 & 21.21 & 19.36 & 18.38 & 17.89 & 17.50 \\
\hline 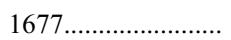 & 53,148 & 262 & 226.092130 & 43.630090 & 0.1931 & 0.3080 & 22.36 & 20.87 & 19.45 & 18.88 & 18.46 \\
\hline $1746 \ldots \ldots \ldots \ldots \ldots \ldots \ldots \ldots$ & 53,062 & 533 & 155.484860 & 14.117880 & 0.1402 & 0.2469 & 22.03 & 20.37 & 19.02 & 18.46 & 18.12 \\
\hline
\end{tabular}

quality of the pairs. The ideal occulting pair - spiral over ellipticalprovides the best estimate of the dust content of a spiral disk (Fig. 1). An additional advantage of this SDSS sample is a high separation in redshift $(\Delta z)$ between the background and foreground galaxy; there are fewer issues with the scatter of background light and interacting pairs.

This SDSS sample has selection properties more like what we are likely to get in the high- $z$ fields than our carefully culled previous overlap sample (e.g., carefully culled to reduce the incidence of galaxies with asymmetry issues) and this, while the bulk measurements will have larger errors, is perhaps a better starting point if we want to look for evolution of the disk extinction. Also, this technique, while very likely suboptimal for some individual marginal cases where an analysis "by hand" could do better, is more like what one can try on the large samples of poorly resolved systems at higher redshift $(z>0.3)$.

\section{SAMPLE SELECTION}

Warren et al. (1996) and Bolton et al. (2004) describe a technique to select high-redshift spiral galaxies lensed by a foreground elliptical galaxy from the SDSS DR4 spectroscopic sample for HST snapshot follow-up (Koopmans 2004; Bolton 2005). They select red, absorption-dominated spectra - typical for ellipticalsthat also display multiple high-redshift emission lines associated with the background lensed spiral galaxy (Bolton et al. 2006; Treu et al. 2006; Koopmans et al. 2006; Gavazzi et al. 2007). It is a straightforward matter to implement the reverse of this algorithm to select absorption-dominated spectra with emission lines at lower redshifts to find spiral disks with a bright background elliptical behind them.

Objects were selected from the fourth data release of the SDSS spectra if they met the following criteria:

1. The redshift is successfully found by the Princeton 1D spectral pipeline. ${ }^{4}$

2. The object is classified spectroscopically as a galaxy by this pipeline: the $\chi^{2}$ fit is better for a galaxy spectrum than a star or QSO.

3. Rest-frame $\mathrm{H} \alpha$ equivalent width less than $4 \AA$ to select ellipticals.
4. Redshift less than 0.4 to enable the $\mathrm{H} \alpha$ equivalent width cut in the optical band.

5. At least three of five lines ([O II $], \mathrm{H} \beta,[\mathrm{O}$ III $] \lambda 4960$, [O III] $\lambda 5008$, and $\mathrm{H} \alpha$ ) are detected at a redshift greater than 0.01 but less than the elliptical's redshift.

The reversed algorithm yielded 118 candidate occulting pairs in the SDSS DR4 spectral sample. Both galaxies are within the $3^{\prime \prime}$ aperture of the SDSS fiber, ensuring a small angular separation of the occulting pair members. In addition, we have visually verified the suitability of each pair and we picked only those that have the ideal pair geometry (Fig. 1). The resulting sample is 83 ideal pairs. The Sloan spectroscopy limits us effectively to galaxies with redshifts less than $0.4(z<0.4)$, and most of the spirals are closer than $0.3(z<0.3)$. Figure 3 shows the distribution of redshifts for the foreground and background galaxies. The majority of the foreground galaxies are nearby $(z<0.1)$. The SDSS DR4 spectral catalog information is listed in Table 1 . These spirals, backlit by a bright symmetric elliptical, enable us to measure the extinction to the highest degree of accuracy possible with this technique.

The SDSS (York et al. 2000) has mapped one-quarter of the entire sky, mainly around the north Galactic cap, in five bands, $u^{\prime}, g^{\prime}, r^{\prime}, i^{\prime}, z^{\prime}$ (Smith et al. 2002; Fukugita et al. 1996). SDSS imaging is obtained using a drift-scanning mosaic CCD camera (Gunn et al. 1998) with a pixel size of $0.396^{\prime \prime}$. We obtained all five bands of the night sky ("corrected frames") in fits format using the SDSS SkyServer DR5. ${ }^{5}$ The corrected frames, having been bias-subtracted, flat-fielded, and purged of bright stars are stored at SDSS in integer format to save disk space. The pixel values get randomized appropriately before being rounded to make sure that the statistics of the background counts are what they should be. An additional offset (SOFTBIAS) of 1000 counts is added to each pixel to avoid negative pixel values and should be subtracted together with the sky value. We used only single SDSS scans for our analysis (no pair was on the dividing line between scans).

\section{FIT TO THE OCCULTING PAIR}

The uniform approach to the SDSS images of these pairs is to fit the images with the central $x$ and $y$ position of the foreground

\footnotetext{
${ }^{4}$ See http://spectro.princeton.edu.
}

\footnotetext{
${ }^{5}$ See http://cas.sdss.org/dr5/en/tools/chart/chart.asp.
} 
TABLE 2

The Optical Depths of the SDSS Filters

\begin{tabular}{|c|c|c|c|c|c|c|c|c|c|c|c|}
\hline Plate & MJD & Fiber & $z_{\mathrm{fg}}$ & $\mathrm{z}_{\mathrm{bg}}$ & $R$ & Incl. & $\tau_{u}$ & $\tau_{g}$ & $\tau_{r}$ & $\tau_{i}$ & $\tau_{z}$ \\
\hline $267 \ldots \ldots \ldots \ldots$ & 51,608 & 499 & 0.04 & 0.09 & $\ldots$ & 0.0 & $\ldots$ & $0.44(3.65)$ & $-0.01(26.35)$ & .. & $\ldots(1.84)$ \\
\hline $273 \ldots \ldots \ldots \ldots$ & 51,957 & 323 & 0.11 & 0.18 & $\ldots$ & 0.0 & $\ldots$ & $\ldots$ & .. & $\ldots$ & $\ldots$ \\
\hline $275 \ldots \ldots \ldots \ldots$ & 51,910 & 233 & 0.03 & 0.09 & $0.81(0.13)$ & 39.0 & $-0.39(2.39)$ & $0.21(3.61)$ & $0.10(11.02)$ & $0.06(2.14)$ & $0.23(1.79)$ \\
\hline $281 \ldots \ldots \ldots \ldots$ & 51,614 & 624 & 0.05 & 0.20 & $\ldots$ & 0.0 & $\ldots$ & $\ldots$ & $\ldots$ & $\ldots$ & $\ldots$ \\
\hline $282 \ldots \ldots \ldots \ldots$ & 51,658 & 120 & 0.07 & 0.26 & $1.82(0.27)$ & 61.7 & $\ldots$ & $0.10(2.19)$ & $0.01(3.85)$ & $0.04(5.93)$ & $0.31(1.82)$ \\
\hline $285 \ldots \ldots \ldots \ldots$ & 51,930 & 15 & 0.02 & 0.14 & $\ldots$ & 0.0 & $\ldots$ & $\ldots$ & $\ldots$ & $\ldots$ & $0.0(18.51)$ \\
\hline 313............... & 51,673 & 546 & 0.04 & 0.30 & $\ldots$ & 0.0 & $\ldots$ & $-4.76(1.94)$ & $\ldots$ & $\ldots$ & $\ldots$ \\
\hline $367 \ldots \ldots \ldots \ldots$ & 51,997 & 222 & 0.08 & 0.14 & $\ldots$ & 0.0 & $\ldots$ & $\ldots$ & $\ldots$ & $\ldots$ & $0.0(2.63)$ \\
\hline $403 \ldots \ldots \ldots \ldots$ & 51,871 & 586 & 0.14 & 0.17 & $\ldots$ & 0.0 & $\ldots$ & $\ldots$ & $\ldots$ & $\ldots$ & $0.0(2.29)$ \\
\hline $408 \ldots \ldots \ldots \ldots$ & 51,821 & 336 & 0.07 & 0.12 & $1.11(0.45)$ & 27.7 & $\ldots$ & $\ldots$ & $0.20(4.11)$ & $-0.02(1.86)$ & $-0.12(2.78)$ \\
\hline $435 \ldots \ldots \ldots \ldots$ & 51,882 & 118 & 0.23 & 0.26 & $\ldots$ & 0.0 & $\ldots$ & $\ldots$ & $\ldots$ & $\ldots$ & $0.0(1.55)$ \\
\hline $436 \ldots \ldots \ldots \ldots$ & 51,883 & 638 & 0.04 & 0.36 & $1.63(0.27)$ & 57.0 & $\ldots$ & $-0.13(4.92)$ & $0.42(2.31)$ & $0.19(1.88)$ & $0.50(2.66)$ \\
\hline $440 \ldots \ldots \ldots \ldots$ & 51,885 & 370 & 0.18 & 0.25 & $\ldots$ & 0.0 & $\ldots$ & $\ldots$ & $\ldots$ & $\ldots$ & $0.0(1.67)$ \\
\hline $464 \ldots \ldots \ldots \ldots$ & 51,908 & 211 & 0.12 & 0.20 & $\ldots$ & 0.0 & $\ldots$ & $0.98(3.12)$ & $\ldots$ & $\ldots$ & $\ldots$ \\
\hline $474 \ldots \ldots \ldots \ldots$ & 52,000 & 548 & 0.21 & 0.30 & $\ldots$ & 0.0 & $\ldots$ & $\ldots$ & $\ldots$ & $\ldots$ & $\ldots$ \\
\hline $484 \ldots \ldots \ldots \ldots$ & 51,907 & 616 & 0.16 & 0.25 & $\ldots$ & 0.0 & $\ldots$ & $-0.83(6.27)$ & $-0.13(2.13)$ & $\ldots$ & $\ldots$ \\
\hline $487 \ldots \ldots \ldots \ldots$ & 51,943 & 305 & 0.12 & 0.25 & $3.48(0.39)$ & 59.5 & $\ldots$ & $0.37(6.54)$ & $-0.23(7.13)$ & $-0.20(5.69)$ & $-0.23(\ldots)$ \\
\hline $497 \ldots \ldots \ldots \ldots$ & 51,989 & 73 & 0.20 & 0.40 & $1.12(0.42)$ & 38.6 & $\ldots$ & $\ldots$ & $-0.05(24.50)$ & $0.23(2.12)$ & $-1.66(\ldots)$ \\
\hline $499 \ldots \ldots \ldots \ldots$ & 51,988 & 152 & 0.11 & 0.29 & $1.81(0.64)$ & 49.7 & $\ldots$ & $\ldots$ & $0.0(2.54)$ & $0.76(61.34)$ & $0.0(1.50)$ \\
\hline $511 \ldots \ldots \ldots \ldots$ & 52,636 & 313 & 0.13 & 0.27 & $0.81(0.37)$ & 52.8 & $\ldots$ & $\ldots$ & $\ldots$ & $0.90(4.77)$ & $0.50(2.29)$ \\
\hline $512 \ldots \ldots \ldots \ldots$ & 51,992 & 302 & 0.05 & 0.23 & $1.26(0.29)$ & 44.3 & $\ldots$ & $-0.32(1.72)$ & $0.12(2.86)$ & $0.03(3.82)$ & $1.50(5.58)$ \\
\hline $529 \ldots \ldots \ldots \ldots$ & 52,025 & 408 & 0.02 & 0.17 & $\ldots$ & 0.0 & $\ldots$ & $\ldots$ & $\ldots$ & $\ldots$ & $0.0(1.70)$ \\
\hline $550 \ldots \ldots \ldots \ldots$ & 51,959 & 624 & 0.17 & 0.20 & $\ldots$ & 0.0 & $\ldots$ & $0.18(78.96)$ & $\ldots$ & $\ldots$ & $\ldots$ \\
\hline $628 \ldots \ldots \ldots \ldots$ & 52,083 & 262 & 0.03 & 0.12 & $\ldots$ & 0.0 & $\ldots$ & $\ldots$ & $\ldots$ & $\ldots$ & $0.0(2.31)$ \\
\hline $629 \ldots \ldots \ldots \ldots$ & 52,051 & 26 & 0.12 & 0.35 & $0.85(0.34)$ & 40.7 & $\ldots$ & $\ldots$ & $0.09(2.35)$ & $-0.20(2.18)$ & $1.43(26.06)$ \\
\hline 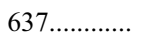 & 52,174 & 334 & 0.09 & 0.18 & $1.06(0.46)$ & 48.6 & $\ldots$ & $0.0(1.67)$ & $0.0(4.10)$ & $1.10(2.86)$ & $0.0(3.29)$ \\
\hline $671 \ldots \ldots \ldots \ldots$ & 52,206 & 347 & 0.04 & 0.28 & $4.23(0.14)$ & 24.2 & $\ldots$ & $0.0(6.47)$ & $-0.01(2.07)$ & 1.89 (13.63) & $1.53(\ldots)$ \\
\hline $765 \ldots \ldots \ldots \ldots$ & 52,254 & 179 & 0.04 & 0.21 & $\ldots$ & 0.0 & $\ldots$ & $\ldots$ & $\ldots$ & $\ldots$ & $\ldots$ \\
\hline $782 \ldots \ldots \ldots \ldots . .$. & 52,320 & 306 & 0.15 & 0.21 & $\ldots$ & 0.0 & $\ldots$ & $0.27(1.36)$ & $\ldots$ & $\ldots$ & $\ldots$ \\
\hline $790 \ldots \ldots \ldots \ldots$ & 52,441 & 527 & 0.03 & 0.14 & $1.83(0.30)$ & 67.3 & $0.0(2.24)$ & $-0.16(4.21)$ & $\ldots$ & $\ldots$ & $-0.15(2.43)$ \\
\hline $792 \ldots \ldots \ldots \ldots$ & 52,353 & 432 & 0.14 & 0.20 & $1.76(0.41)$ & 23.1 & $\ldots$ & $0.13(2.70)$ & $-0.03(2.03)$ & $0.06(2.04)$ & $-0.16(1.81)$ \\
\hline $808 \ldots \ldots \ldots \ldots . . .$. & 52,556 & 39 & 0.21 & 0.37 & $\ldots$ & 0.0 & $\ldots$ & $\ldots$ & $\ldots$ & $\ldots$ & $\ldots$ \\
\hline $845 \ldots \ldots \ldots \ldots$ & 52,381 & 270 & 0.07 & 0.32 & $\ldots$ & 0.0 & $\ldots$ & $\ldots$ & $\ldots$ & $\ldots$ & $\ldots$ \\
\hline $848 \ldots \ldots \ldots \ldots$ & 52,669 & 602 & 0.05 & 0.22 & $2.22(0.19)$ & 42.0 & $\ldots$ & $\ldots$ & $-0.07(1.91)$ & $\ldots$ & $0.60(2.64)$ \\
\hline $860 \ldots \ldots \ldots . .$. & 52,319 & 451 & 0.04 & 0.16 & $1.66(0.23)$ & 32.5 & $0.0(2.33)$ & $0.29(0.79)$ & $0.41(1.02)$ & $0.23(2.62)$ & $0.31(2.04)$ \\
\hline $864 \ldots \ldots \ldots \ldots$ & 52,320 & 91 & 0.04 & 0.26 & $\ldots$ & 0.0 & $\ldots$ & $0.24(1.81)$ & $-0.24(19.63)$ & $-0.05(2.01)$ & $\ldots$ \\
\hline $877 \ldots \ldots \ldots \ldots$ & 52,353 & 458 & 0.04 & 0.06 & $\ldots$ & 0.0 & $\ldots$ & $\ldots$ & $\ldots$ & $\ldots$ & $\ldots$ \\
\hline $882 \ldots \ldots \ldots \ldots . .$. & 52,370 & 122 & 0.06 & 0.18 & $\ldots$ & 0.0 & $\ldots$ & $\ldots$ & $\ldots$ & $\ldots$ & $0.0(68.77)$ \\
\hline $883 \ldots \ldots \ldots \ldots . . .$. & 52,430 & 366 & 0.05 & 0.13 & $\ldots$ & 0.0 & $\ldots$ & $0.11(4.58)$ & $-0.07(2.56)$ & $-0.03(6.10)$ & ... \\
\hline $884 \ldots \ldots \ldots \ldots$ & 52,374 & 242 & 0.04 & 0.17 & $\ldots$ & 0.0 & $\ldots$ & $\ldots$ & $\ldots$ & $\ldots$ & $\ldots(3.84)$ \\
\hline $889 \ldots \ldots \ldots \ldots$ & 52,663 & 408 & 0.06 & 0.16 & $\ldots$ & 0.0 & $\ldots$ & $\ldots$ & $\ldots$ & $\ldots$ & $\ldots$ \\
\hline $896 \ldots \ldots \ldots \ldots$ & 52,592 & 463 & 0.03 & 0.11 & $2.49(0.19)$ & 45.8 & $0.0(123.79)$ & $0.37(10.70)$ & $0.16(5.94)$ & $0.09(47.10)$ & $0.16(\ldots)$ \\
\hline $913 \ldots \ldots \ldots \ldots$ & 52,433 & 151 & 0.08 & 0.16 & $\ldots$ & 0.0 & $\ldots$ & $0.10(1.79)$ & $-0.02(2.56)$ & $\ldots$ & $\ldots(2.36)$ \\
\hline $931 \ldots \ldots \ldots \ldots . . . .$. & 52,619 & 84 & 0.18 & 0.28 & $\ldots$ & 0.0 & $\ldots$ & .. & $\ldots$ & $\ldots$ & $\ldots$ \\
\hline $958 \ldots \ldots \ldots \ldots$ & 52,410 & 194 & 0.15 & 0.31 & $4.49(0.81)$ & 36.3 & $\ldots$ & $\ldots$ & $-0.38(2.72)$ & $-0.62(1.29)$ & $0.46(\ldots)$ \\
\hline $970 \ldots \ldots \ldots \ldots$ & 52,413 & 408 & 0.30 & 0.38 & $\ldots$ & 0.0 & $\ldots$ & $\ldots$ & $\ldots$ & $\ldots$ & $\ldots(3.52)$ \\
\hline $980 \ldots \ldots \ldots \ldots$ & 52,431 & 300 & 0.13 & 0.29 & $1.49(0.20)$ & 18.1 & $\ldots$ & $-0.67(2.25)$ & $-0.07(1.48)$ & $-0.21(1.76)$ & $-0.40(\ldots)$ \\
\hline $1000 \ldots \ldots \ldots$ & 52,643 & 337 & 0.09 & 0.32 & $\ldots$ & 0.0 & $\ldots$ & $\ldots$ & $0.27(2.88)$ & $0.22(1860.97)$ & \\
\hline $1006 \ldots \ldots \ldots$ & 52,708 & 624 & 0.05 & 0.13 & $1.86(0.24)$ & 53.2 & $\ldots$ & $0.0(1.54)$ & $0.40(1.60)$ & $0.28(2.76)$ & $0.37(1.88)$ \\
\hline $1007 \ldots \ldots \ldots . .$. & 52,706 & 519 & 0.05 & 0.20 & $\ldots$ & 0.0 & $\ldots$ & $\ldots$ & $\ldots$ & $\ldots$ & \\
\hline $1160 \ldots \ldots \ldots$ & 52,674 & 468 & 0.24 & 0.29 & $\ldots$ & 0.0 & $\ldots$ & $\ldots$ & $\ldots$ & $\cdots$ & $0.0(4.23)$ \\
\hline $1215 \ldots \ldots \ldots$ & 52,725 & 288 & 0.27 & 0.33 & $\ldots$ & 0.0 & $\ldots$ & $-0.01(1.73)$ & $\ldots$ & $\ldots$ & $0.0(1.96)$ \\
\hline $1230 \ldots \ldots \ldots$ & 52,672 & 639 & 0.05 & 0.16 & $1.19(0.37)$ & 60.9 & $0.0(2.30)$ & $0.18(2.21)$ & $0.27(2.85)$ & $0.11(125.74)$ & $-0.92(\ldots)$ \\
\hline $1235 \ldots \ldots \ldots . .$. & 52,734 & 164 & 0.10 & 0.33 & $0.88(0.24)$ & 61.2 & $\ldots$ & $-0.03(1.94)$ & $-0.20(3.08)$ & $0.13(9.64)$ & $-0.19(\ldots)$ \\
\hline $1269 \ldots \ldots \ldots$ & 52,937 & 485 & 0.03 & 0.19 & $\ldots$ & 0.0 & $\ldots$ & $0.01(5.78)$ & $\ldots$ & $\ldots$ & $\ldots$ \\
\hline $1280 \ldots \ldots \ldots . . .$. & 52,738 & 125 & 0.08 & 0.21 & $\ldots$ & 0.0 & $\ldots$ & .. & $\ldots$ & $\ldots$ & $\ldots$ \\
\hline $1282 \ldots \ldots \ldots$ & 52,759 & 630 & 0.12 & 0.28 & $0.57(0.26)$ & 43.7 & $\ldots$ & $0.0(1.93)$ & $0.44(207.10)$ & $0.26(19.18)$ & $-0.17(1.72)$ \\
\hline $1310 \ldots \ldots \ldots$ & 53,033 & 202 & 0.11 & 0.18 & $\ldots$ & 0.0 & $\ldots$ & $\ldots$ & $\ldots$ & $\ldots$ & $\ldots$ \\
\hline $1317 \ldots \ldots \ldots$ & 52,765 & 9 & 0.10 & 0.20 & $\ldots$ & 0.0 & $\ldots$ & $\ldots$ & $\ldots$ & $\ldots$ & $\ldots$ \\
\hline $1321 \ldots \ldots \ldots$ & 52,764 & 461 & 0.15 & 0.22 & $2.14(0.41)$ & 35.4 & $\ldots$ & $-0.50(32.41)$ & $-0.03(3.15)$ & $0.20(1.73)$ & $\ldots$ \\
\hline $1324 \ldots \ldots \ldots$ & 53,088 & 50 & 0.04 & 0.23 & $1.97(0.32)$ & 68.4 & $\ldots$ & $-0.19(2.20)$ & $-0.03(2.16)$ & $-0.12(2.98)$ & $0.04(\ldots)$ \\
\hline $1325 \ldots \ldots \ldots . .$. & 52,762 & 313 & 0.12 & 0.21 & $\ldots$ & 0.0 & $\ldots$ & $-0.02(2.39)$ & $\ldots$ & $\ldots$ & $\ldots$ \\
\hline $1327 \ldots \ldots \ldots$ & 52,781 & 252 & 0.08 & 0.14 & $\ldots$ & 0.0 & $\ldots$ & $\ldots$ & $\ldots$ & $\ldots$ & $\ldots$ \\
\hline $1332 \ldots \ldots \ldots$ & 52,781 & 469 & 0.28 & 0.32 & $5.83(0.64)$ & 23.2 & $\ldots$ & $\ldots$ & $-1.09(2.08)$ & $-1.25(2.37)$ & $-1.29(1.39)$ \\
\hline $1363 \ldots \ldots \ldots$ & 53,053 & 104 & 0.18 & 0.27 & $1.12(0.38)$ & 55.6 & $\ldots$ & $0.0(1.55)$ & $-0.05(9.05)$ & $0.17(7.07)$ & $0.21(\ldots)$ \\
\hline
\end{tabular}


TABLE 2-Continued

\begin{tabular}{|c|c|c|c|c|c|c|c|c|c|c|c|}
\hline Plate & MJD & Fiber & $z_{\mathrm{fg}}$ & $z_{\mathrm{bg}}$ & $R$ & Incl. & $\tau_{u}$ & $\tau_{g}$ & $\tau_{r}$ & $\tau_{i}$ & $\tau_{z}$ \\
\hline $1373 \ldots \ldots \ldots$ & 53,063 & 255 & 0.03 & 0.13 & & 0.0 & $0.0(2.30)$ & $\ldots$ & & & . \\
\hline $1390 \ldots \ldots \ldots$ & 53,142 & 385 & 0.13 & 0.29 & $2.08(0.49)$ & 26.1 & $\ldots$ & $\ldots$ & $0.07(2.41)$ & $0.05(1.68)$ & $0.07(\ldots)$ \\
\hline $1416 \ldots \ldots \ldots$ & 52,875 & 490 & 0.07 & 0.19 & $2.18(1.17)$ & 14.4 & $\ldots$ & $-0.03(1.77)$ & $\ldots$ & $\ldots$ & $0.0(2.92)$ \\
\hline $1423 \ldots \ldots \ldots$ & 53,167 & 528 & 0.20 & 0.33 & $1.73(0.50)$ & 42.2 & $\ldots$ & $\ldots$ & $-0.54(1.35)$ & $-0.18(1.77)$ & $-0.30(\ldots)$ \\
\hline $1429 \ldots \ldots \ldots$ & 52,990 & 336 & 0.07 & 0.15 & $1.25(0.16)$ & 42.7 & $0.0(2.61)$ & $0.0(2.78)$ & 0.67 (1.63) & $0.06(2.11)$ & $1.54(9.57)$ \\
\hline $1604 \ldots \ldots \ldots . .$. & 53,078 & 459 & 0.08 & 0.17 & $0.82(0.43)$ & 42.2 & $\ldots$ & $0.94(6.10)$ & $0.49(1.32)$ & $1.61(1.98)$ & \\
\hline $1607 \ldots \ldots \ldots$ & 53,083 & 48 & 0.08 & 0.15 & $\ldots$ & 0.0 & $\ldots$ & $-0.63(6.30)$ & $\ldots$ & $\ldots$ & $0.0(1.39)$ \\
\hline $1618 \ldots \ldots \ldots . . . .$. & 53,116 & 285 & 0.04 & 0.17 & $\ldots$ & 0.0 & $\ldots$ & $\ldots$ & $\ldots$ & $\ldots$ & $0.0(2.32)$ \\
\hline $1620 \ldots \ldots \ldots$ & 53,137 & 175 & 0.07 & 0.10 & $1.00(0.21)$ & 47.7 & $0.0(8.61)$ & $-0.23(1.69)$ & $0.05(5.04)$ & $-0.17(23.12)$ & $-0.12(\ldots)$ \\
\hline $1677 \ldots \ldots \ldots$ & 53,148 & 262 & 0.19 & 0.31 & $\ldots$ & 0.0 & $\ldots$ & $\ldots$ & $\ldots$ & $\ldots$ & $\ldots$ \\
\hline $1746 \ldots \ldots \ldots$ & 53,062 & 533 & 0.14 & 0.25 & $1.21(0.38)$ & 50.1 & $\ldots$ & $0.39(4.83)$ & $0.80(1.64)$ & $0.36(1.92)$ & $-0.23(\ldots)$ \\
\hline
\end{tabular}

Notes.-These values have not been corrected for inclination. Uncertainties are in parentheses.

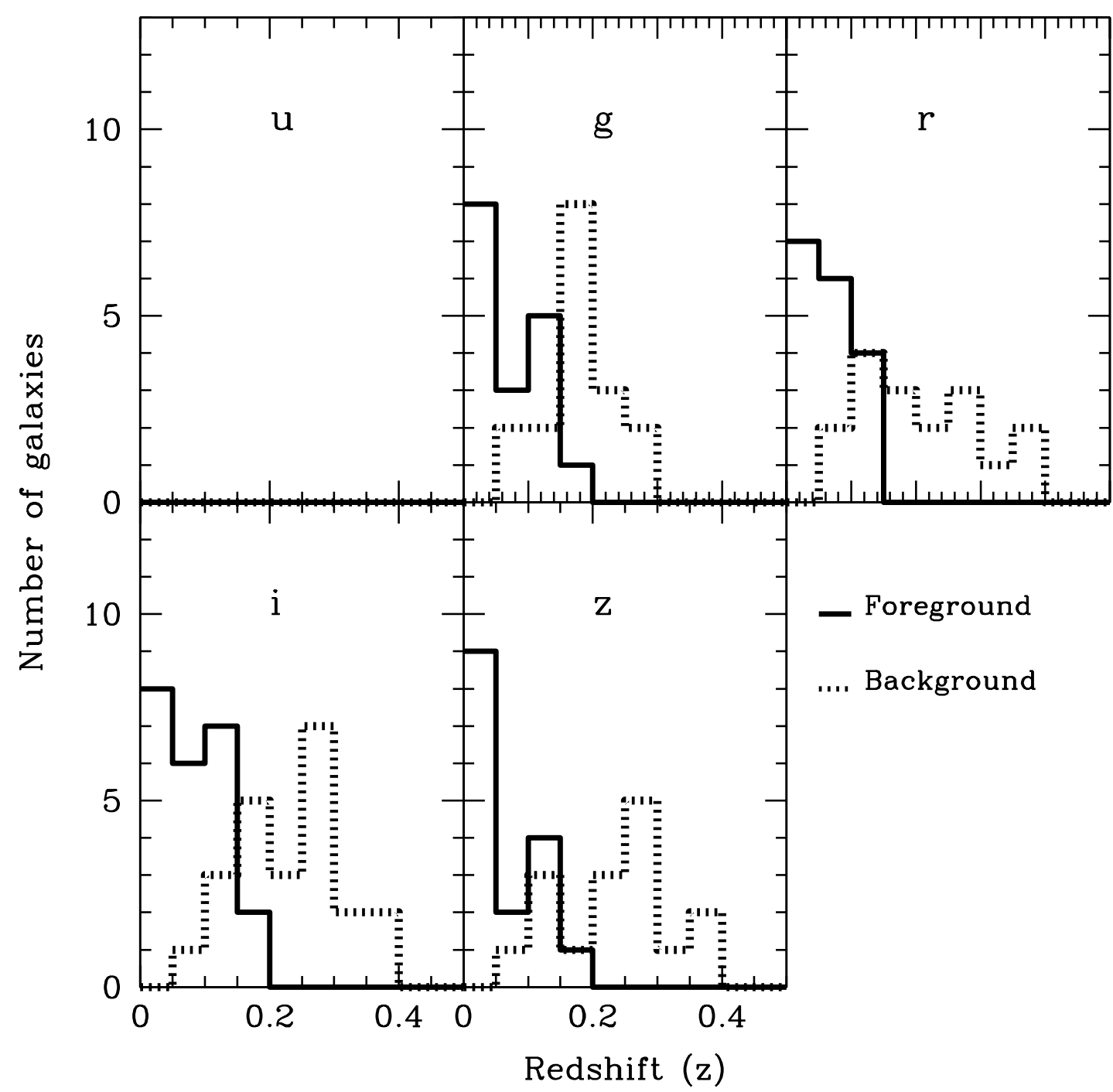

FIG. 4.- Histogram of the redshift distribution of both the background and foreground galaxies in pairs for which a successful fit is found. No good fits are obtained for the $u$ band, and only disks closer than $z=0.2$ could be fitted. 


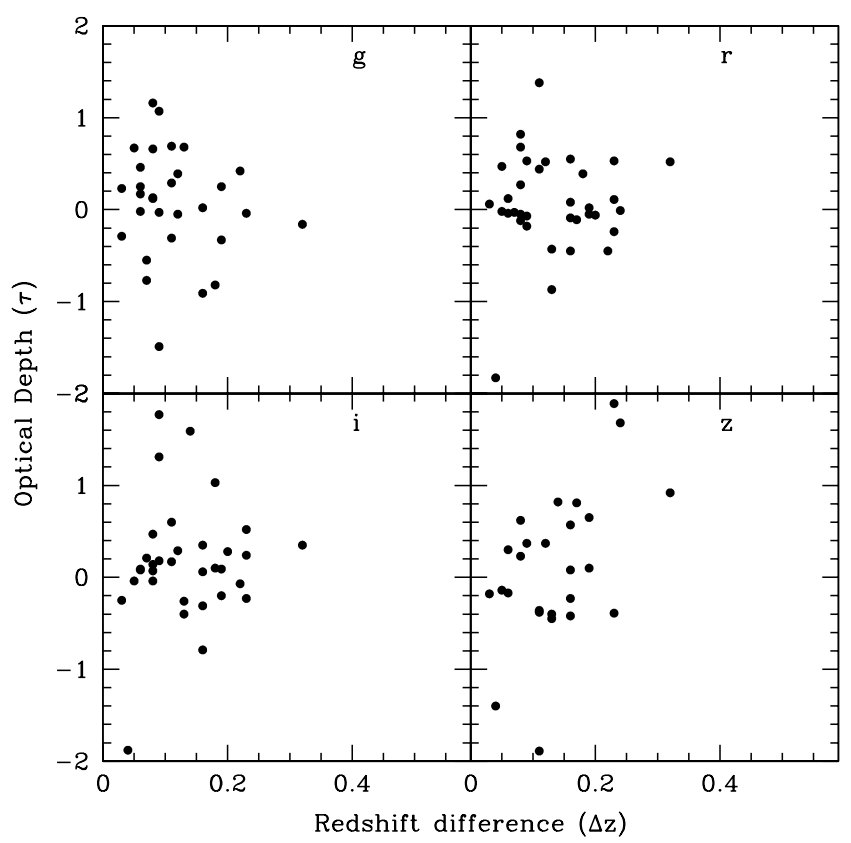

FIG. 5.-Plots of the redshift distance between foreground and background galaxy and the derived optical depth in the visual aperture in the SDSS for which meaningful opacities can be obtained. There appears to be no systematic relation between the distance between the pair members and the optical depth inferred.

and background galaxy as free parameters, as well as the angle of rotation of both galaxies: six free parameters in total $\left(x_{\mathrm{fg}}, y_{\mathrm{fg}}\right.$, $\mathrm{pa}_{\mathrm{fg}}, x_{\mathrm{bg}}, y_{\mathrm{bg}}$, and $\left.\mathrm{pa}_{\mathrm{bg}}\right)$. Figure 2 shows a schematic of the fit. The best-fit criteria is a minimal residual image, the original image with the two rotated galaxies subtracted. We used a new fit for each SDSS filter. Because the image is used to model itself, we do not need to take the SDSS point-spread function into account.

To identify objects in the field and the members of the pair, we ran Source Extractor version 2.5 (Bertin \& Arnouts 1996). ${ }^{6}$ If both members of the pair are separated and identified in the segmentation by Source Extractor, we can proceed with the fit. We use the assignments of pixels by Source Extractor to mask nonpair objects. A $100 \times 100$ pixel postage stamp is cut from the SDSS scan around the pair and used for the further fit with the IDL routine mpfit2dfun. The mask for each pair member is padded by smoothing it with a 3 pixel wide boxcar. Typical galaxy sizes are several hundred pixels. We use the padded masks to determine which pixels are to be used for the fit. The model subtracted from the original image is the foreground galaxy rotated, plus the background galaxy rotated with the sky value subtracted from the original image. The Source Extractor catalog used to make the segmentation image also provides the effective radius, ellipticity, and flux for both the foreground galaxy and the background galaxy. The ellipticity is converted into an inclination estimate $[i=\arctan (B / A)]$.

We apply two apertures to the extinction map based on the fits, each $5 \times 5$ pixels. In the $g$-band images, we visually identified a good position for an aperture. An additional aperture position can be identified in the extinction map by using the point of maximum extinction as the center of the second aperture. Extinction values for the visual aperture are presented in Table 2 for the SDSS filters. The disk opacities have been corrected for the inclination $(\times \cos i)$.

\footnotetext{
${ }^{6}$ See also the user manual, Holwerda (2005)
}

The inclusion of an automatically selected aperture position was to test whether or not this method can be easily automated to apply to large samples of near pairs. It appears that a visual placement of the aperture remains optimal.

The fit fails in cases in which Source Extractor did not segment the pair into two separate objects or when there is no signal in the visual aperture. Negative extinction values occur when the galaxies are significantly asymmetric but the fit does not explicitly fail. We include these negative opacity values because it gives an indication of the dominant remaining uncertainty in these measurements.

Figure 4 shows the redshift distributions for pairs with a successful fit. An opacity measurement of a spiral disk from SDSS images is feasible for disks closer than $z=0.2$. The fit fails for more distant disks as the pair cannot be resolved into separate objects. Figure 5 shows the optical depth as a function of pair separation in redshift. There is no systematic effect which confimrs that these pairs are well separated.

\section{RESULTS}

The optical depth value of a spiral disks depends a great many factors, such as radius, arm presence, Hubble type, and possibly disk luminosity. In this section we explore some relations between disk opacities and other disk characteristics, such as radius, disk luminosity, and surface brightness. Finally, we present radial plots based on stacked SDSS filters together to improve the signalto-noise ratio $(\mathrm{S} / \mathrm{N})$.

\subsection{Radial Profiles}

Figure 6 shows the radial profiles based on the visual apertures for all five SDSS filters $\left(g^{\prime}, r^{\prime}, i^{\prime}, u^{\prime}, z^{\prime}\right)$ and split up into three redshift ranges $(z<0.05,0.05<z<0.1$, and $z>0.1)$. Figure 7 shows the same plot for the automated aperture. The increase of scatter in the automatic aperture does indicate that the visual aperture is better placed to measure disk opacity. The opacity values have been corrected for inclination $(\times \cos i)$. The $r^{\prime}$ and $i^{\prime}$ filters have the best extinction signal. Notably, the $u^{\prime}$-band fits are all failures, as the early-type background galaxy is too faint in this band and the foreground galaxy possibly too irregular. The reason that the most extinction signal is in the $r^{\prime}$ and $i^{\prime}$ filters is because these are blue enough to exhibit strong dust extinction but red enough to benefit from a symmetric distribution of the light in both galaxies. Bluer filters are more asymmetric due to localized star formation in the foreground galaxy's spiral arms. This is in agreement with $H S T$ WFPC2 results in local galaxies (Keel \& White 2001a, 2001b).

The resolution of the SDSS for these pairs is unfortunately not sufficient to distinguish between arm and disk sections, similar to previous studies in the very local universe. In order to obtain measurements of similar quality, one would require greater resolving power, such as that provided by the HST. The opacity values in Figure 6 are therefore a mix between the arm and disk values.

Similarly, distinguishing Hubble subtypes for these galaxies is equally impossible, and hence the opacities represent also a mix between earlier and later type spirals, which most likely have different opacities (White et al. 2000; Holwerda et al. 2005a).

Because of the many other influences and the small span of the redshift range in Figure 6 it is impossible to conclude whether there is any evolution in the opacity of spiral disks from the singlefilter SDSS data. However, we can conclude that optical disks show evidence of extinction up to twice their effective radius in all SDSS filters except $u$, which is due to low $\mathrm{S} / \mathrm{N}$ from the red background galaxy. 


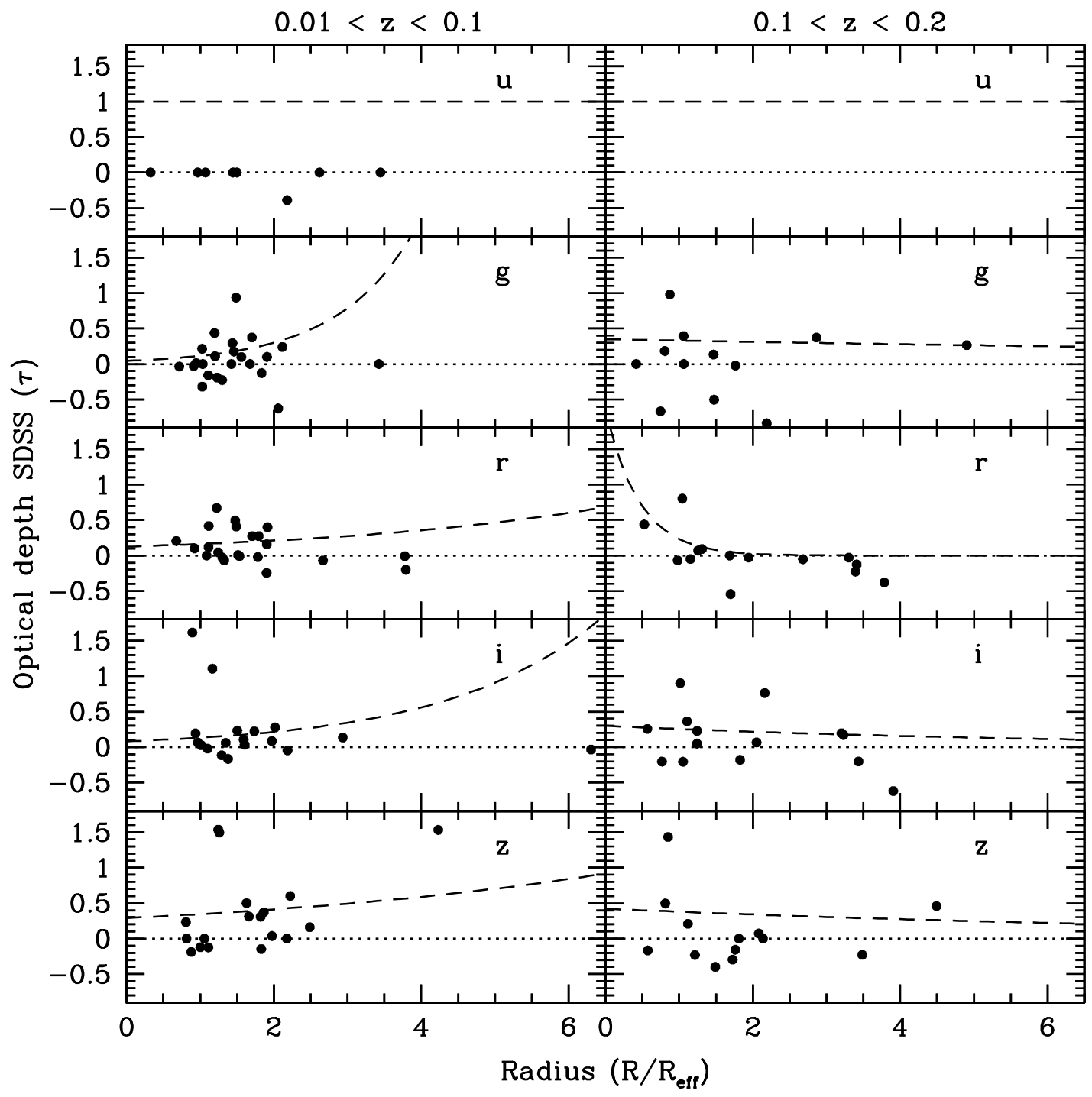

FIG. 6. - Radial opacity plot in all five SDSS filters $\left(u^{\prime}, g^{\prime}, r^{\prime}, i^{\prime}\right.$, and $\left.z^{\prime}\right)$ for the redshift ranges $z=0.01-0.1$ and $0.1-0.2$ for the foreground spiral disks. Radii are scaled by the half-light radius $\left(R_{\text {eff }}\right)$. The opacity measurements are done in the automatically determined aperture. Dashed lines are the exponential fits to the opacity measurements. 


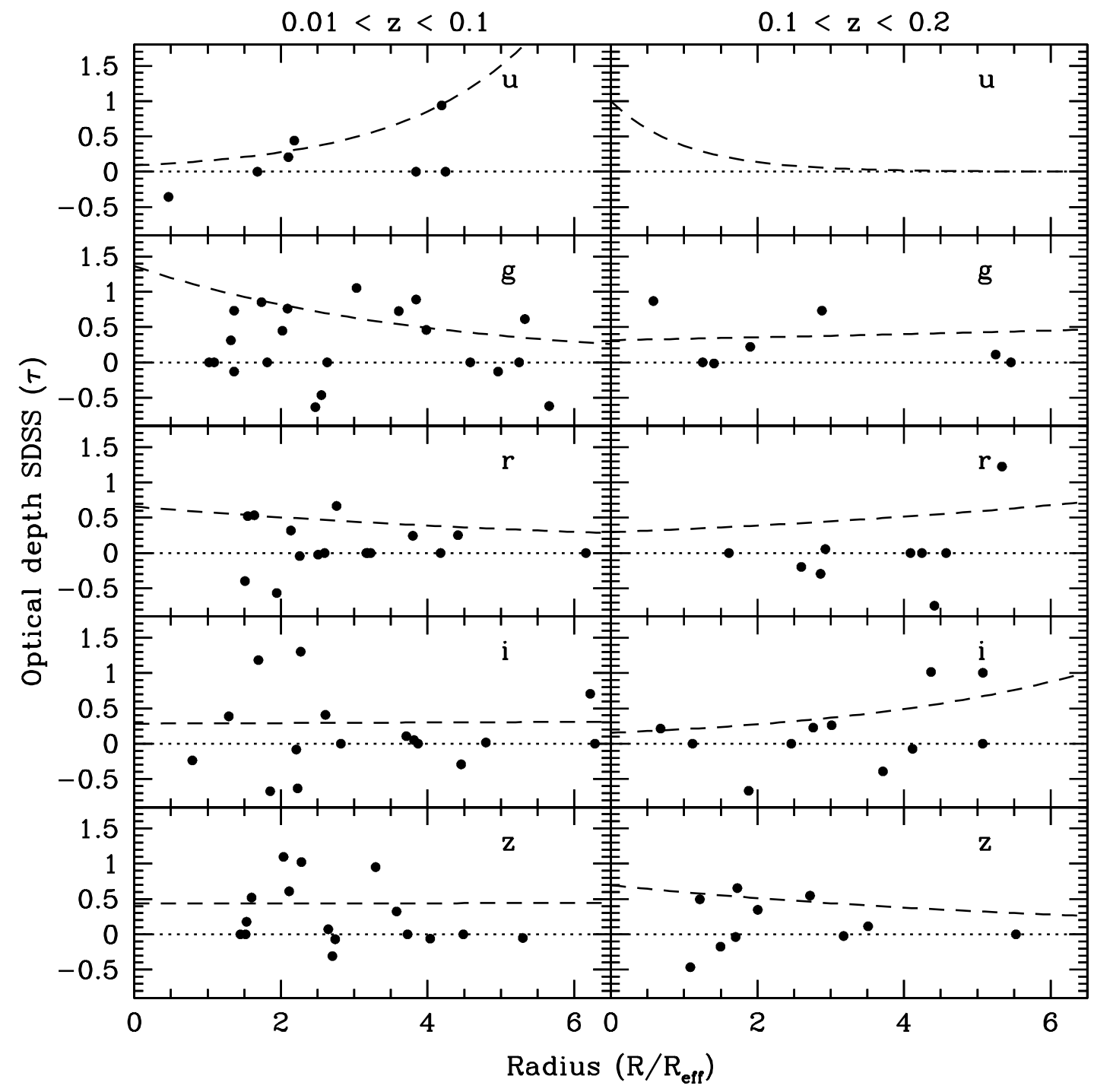

FIG. 7.- Radial opacity plot in all five SDSS filters $\left(u^{\prime}, g^{\prime}, r^{\prime}, i^{\prime}\right.$, and $\left.z^{\prime}\right)$ for the redshift ranges $z=0.01-0.1$ and $0.1-0.2$ for the foreground spiral disks. Radii are scaled by the half-light radius $\left(R_{\text {eff }}\right)$. The opacity measurements are done in the visually determined aperture. Dashed lines are the exponential fits to the opacity measurements. 
TABLE 3

Exponential Fit Values to Optimum Aperture Optical Depths

\begin{tabular}{|c|c|c|c|c|c|c|}
\hline \multirow[b]{2}{*}{ FILTER } & \multicolumn{3}{|c|}{$0.01<z<0.1$} & \multicolumn{3}{|c|}{$0.1<z<0.2$} \\
\hline & $\tau_{0}$ & $h$ & $\mathrm{rms}$ & $\tau_{0}$ & $h$ & $\mathrm{rms}$ \\
\hline$u \ldots \ldots \ldots \ldots \ldots \ldots$ & 0.1 & -1.8 & 0 & $\ldots$ & $\ldots$ & $\ldots$ \\
\hline$g \ldots \ldots \ldots \ldots \ldots$ & 1.4 & 3.9 & 1.0 & 0.3 & -16.7 & 0.6 \\
\hline 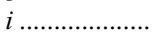 & 0.3 & -70.3 & 1.2 & 0.2 & -3.4 & 0.9 \\
\hline$r \ldots \ldots \ldots \ldots \ldots \ldots$ & 0.7 & 7.6 & 1.2 & 0.3 & -7.2 & 0.8 \\
\hline$z \ldots \ldots \ldots \ldots \ldots \ldots$ & 0.4 & -648.5 & 1.1 & 0.7 & 6.6 & 1.0 \\
\hline
\end{tabular}

\subsection{Exponential Fits}

In Figures 6 and 7 we show the least-squares fit of an exponential disk to the positive points (fit parameters are listed in Tables 3 and 4). The fits are poor and unrealistic (increasing with radius) for many of the SDSS filters. For a better fit, one would need a better $\mathrm{S} / \mathrm{N}$ and be able to distinguish between arm and disk sections.

\subsection{Disk Opacity and Luminosity}

Tully et al. (1998) and Masters et al. (2003) note a relation between disk opacity and overall luminosity. A similar relation for sections of the disks between surface brightness and opacity in spiral arms is noted in Holwerda et al. (2005a, 2005b). Figure 8 shows the relations between the magnitude of the foreground galaxy and disk opacity for all the SDSS filters. No relation can be seen. Figure 9 is the relation between optical depth and surface brightness in the visual aperture. In nearby galaxies there appears to be a relation between the two (Holwerda et al. 2005b), but there is little evidence that brighter disks are also more opaque here.

\subsection{Extinction Law}

With several independent disk opacity measurements in four different filters, one could construct a reddening law for disks at higher redshift. However, the optical depths in the visually identified aperture agree with each other in all four filters (see Fig. 6). As noted by Keel \& White (2001a), disk opacity is effectively gray unless sampled over disk sections smaller than $100 \mathrm{pc}$. The explanation is that the ISM is very clumpy, resulting in a gray extinction law when averaged over larger sections.

\subsection{Stacked Images}

In order to improve the $\mathrm{S} / \mathrm{N}$ of our measurement and given the expected lack of an extinction law, we have stacked various combinations of the SDSS filter images and fit them as a single SDSS filter field. In Table 5 and Figure 11, the results are summarized. The noise does appear somewhat reduced in the radial profiles using $r+i$ stacked fields (Fig. 11). Stacking more fields together or a different combination did not result in any improvement of $\mathrm{S} / \mathrm{N}$ (Table 5).

TABLE 4

Exponential Fit Values to Visual Aperture Optical Depths

\begin{tabular}{|c|c|c|c|c|c|c|}
\hline \multirow[b]{2}{*}{ FILTER } & \multicolumn{3}{|c|}{$0.01<z<0.1$} & \multicolumn{3}{|c|}{$0.1<z<0.2$} \\
\hline & $\tau_{0}$ & $h$ & $\mathrm{rms}$ & $\tau_{0}$ & $h$ & $\mathrm{rms}$ \\
\hline 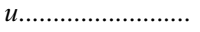 & $\ldots$ & $\ldots$ & $\ldots$ & $\ldots$ & $\ldots$ & $\ldots$ \\
\hline$g \ldots \ldots \ldots \ldots \ldots \ldots \ldots \ldots$ & 0.04 & -1.0 & 1.0 & 0.4 & 17.8 & 0.6 \\
\hline$i \ldots \ldots \ldots \ldots \ldots \ldots \ldots \ldots$ & 0.1 & -2.1 & 1.2 & 0.3 & 6.2 & 0.9 \\
\hline$r \ldots \ldots \ldots \ldots \ldots \ldots \ldots \ldots$ & 0.1 & -3.8 & 1.2 & 2.1 & 0.5 & 0.8 \\
\hline$z \ldots \ldots \ldots \ldots \ldots \ldots \ldots \ldots$ & 0.3 & -5.6 & 1.1 & 0.4 & 9.3 & 1.0 \\
\hline
\end{tabular}

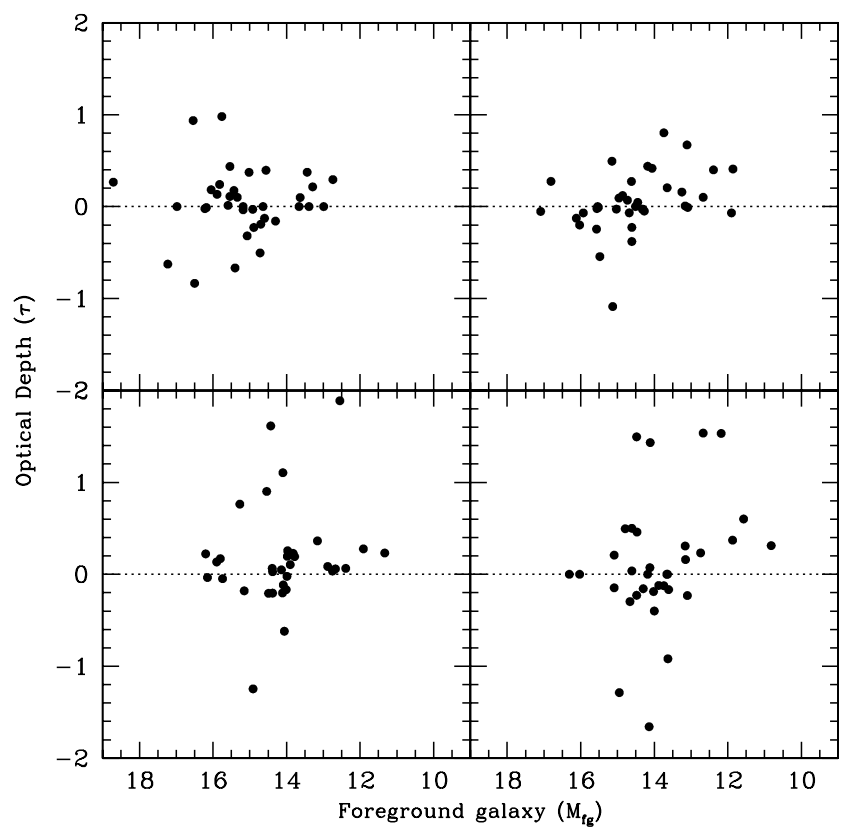

Fig. 8.-Plot of disk optical depth and foreground galaxy magnitude, measured by the Source Extractor run part of the fit. There appears to be little relation between optical depth and disk brightness, probably because other effects - radial distance, arm presence, and Hubble type-dominate the optical depth value. Negative optical depth values occur when there is significant asymmetry in either or both of the members of the occulting pair.

We note that the optical depth measurements in our sample are at radii similar to those by White et al. (2000) and Domingue et al. (2000) (see Fig. 11). As in Figures 6 and 7, we fit an exponential profile to the points (parameters in Table 6), both to the Domingue et al. (2000) points and the $r+i$ opacity points at different redshift. The higher redshift profile fits are extremely flat, with high scale lengths. If we compare the higher redshift profiles to the nearby points, they resemble the arm profile more than the disk. However, much of the profile is determined by opacity measurements nearer to the center of the spiral galaxy.

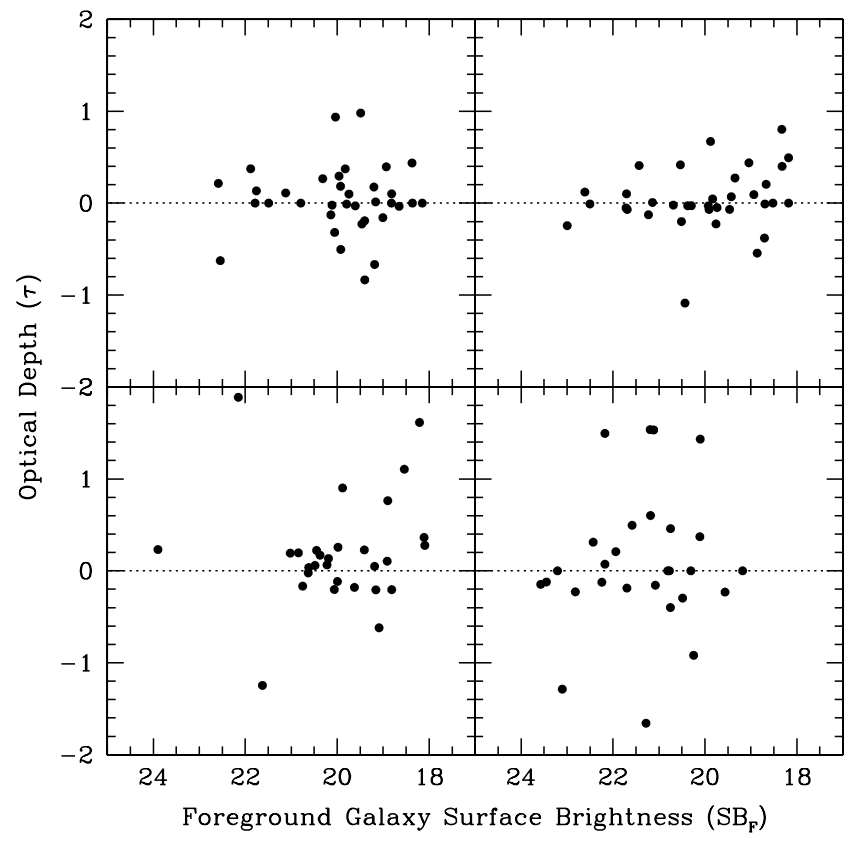

FIG. 9.-Plot of disk optical depth and foreground galaxy surface brightness in the aperture. No relation is evident. 
TABLE 5

The Optical Depths of the Stacked SDSS Filters

\begin{tabular}{|c|c|c|c|c|c|c|c|c|c|c|}
\hline Plate & MJD & Fiber & $z_{\mathrm{fg}}$ & $z_{\mathrm{bg}}$ & $R$ & $\tau_{g r}$ & $\tau_{r i}$ & $\tau_{i z}$ & $\tau_{g r i}$ & $\tau_{\text {griz }}$ \\
\hline $267 \ldots \ldots \ldots \ldots \ldots \ldots \ldots \ldots \ldots$ & 51,608 & 499 & 0.04 & 0.09 & $\ldots$ & $\ldots$ & $\ldots$ & $\ldots$ & $\ldots$ & $-0.13(4.83)$ \\
\hline $273 \ldots \ldots \ldots \ldots \ldots \ldots \ldots \ldots \ldots \ldots \ldots \ldots \ldots \ldots$ & 51,957 & 323 & 0.11 & 0.18 & $\ldots$ & $\ldots$ & $\ldots$ & $\ldots$ & $\ldots$ & $\ldots$ \\
\hline $275 \ldots \ldots \ldots \ldots \ldots \ldots \ldots \ldots$ & 51,910 & 233 & 0.03 & 0.09 & $0.80(0.09)$ & $\ldots$ & $0.30(18.64)$ & $0.05(7.67)$ & $0.31(5.25)$ & $0.05(16.51)$ \\
\hline 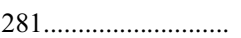 & 51,614 & 624 & 0.05 & 0.20 & $\ldots$ & $\ldots$ & $\ldots$ & $\ldots$ & $\ldots$ & $\ldots$ \\
\hline $282 \ldots \ldots \ldots \ldots \ldots \ldots \ldots \ldots \ldots$ & 51,658 & 120 & 0.07 & 0.26 & $1.52(0.24)$ & $\ldots$ & $-0.03(1.43)$ & $-0.00(1.60)$ & $\ldots$ & $0.00(15.92)$ \\
\hline 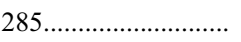 & 51,930 & 15 & 0.02 & 0.14 & $\ldots$ & $\ldots$ & $\ldots$ & $\ldots$ & $\ldots$ & $\ldots$ \\
\hline 313 ........................ & 51,673 & 546 & 0.04 & 0.30 & $\ldots$ & $\ldots$ & $\ldots$ & $\ldots$ & $\ldots$ & $\ldots$ \\
\hline $367 \ldots \ldots \ldots$ & 51,997 & 222 & 0.08 & 0.14 & $\ldots$ & $\ldots$ & $\ldots$ & $\ldots$ & $\ldots$ & $\ldots$ \\
\hline $403 \ldots \ldots \ldots \ldots \ldots \ldots \ldots \ldots$ & 51,871 & 586 & 0.14 & 0.17 & $\ldots$ & $\ldots$ & $\ldots$ & $\ldots$ & $\ldots$ & $\ldots$ \\
\hline $408 \ldots \ldots \ldots \ldots \ldots \ldots \ldots \ldots$ & 51,821 & 336 & 0.07 & 0.12 & $\ldots$ & $\ldots$ & $0.39(1.45)$ & $\ldots$ & $\ldots$ & $\ldots$ \\
\hline $435 \ldots \ldots \ldots \ldots \ldots \ldots \ldots \ldots$ & 51,882 & 118 & 0.23 & 0.26 & $\ldots$ & $\ldots$ & $\ldots$ & $\ldots$ & $\ldots$ & $\ldots$ \\
\hline 436......................... & 51,883 & 638 & 0.04 & 0.36 & $1.00(0.18)$ & $\ldots$ & $0.15(15.17)$ & $0.19(6.96)$ & $\ldots$ & $0.29(8.28)$ \\
\hline $440 \ldots \ldots \ldots \ldots \ldots \ldots \ldots \ldots \ldots$ & 51,885 & 370 & 0.18 & 0.25 & $\ldots$ & $\ldots$ & $\ldots$ & $\ldots$ & $\cdots$ & $\ldots$ \\
\hline 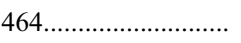 & 51,908 & 211 & 0.12 & 0.20 & $\ldots$ & $\ldots$ & $\ldots$ & $\ldots$ & $\ldots$ & $\ldots$ \\
\hline ..................... & 52,000 & 548 & 0.21 & 0.30 & $\ldots$ & $\ldots$ & $\ldots$ & $\ldots$ & $\ldots$ & $\ldots$ \\
\hline $484 \ldots \ldots$. & 51,907 & 616 & 0.16 & 0.25 & $\ldots$ & $\ldots$ & $\ldots$ & $\ldots$ & $\ldots$ & $\ldots$ \\
\hline 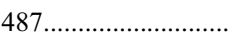 & 51,943 & 305 & 0.12 & 0.25 & $3.51(0.37)$ & $\ldots$ & $-0.24(1.80)$ & $-0.09(4.14)$ & $\ldots$ & $-0.09(3.63)$ \\
\hline 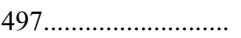 & 51,989 & 73 & 0.20 & 0.40 & $1.27(0.36)$ & $\ldots$ & $0.04(1.79)$ & $0.10(2.47)$ & $\ldots$ & $\ldots$ \\
\hline $499 \ldots \ldots \ldots \ldots \ldots \ldots \ldots \ldots$ & 51,988 & 152 & 0.11 & 0.29 & $\ldots$ & $\ldots$ & $-0.42(1.65)$ & $\ldots$ & $\ldots$ & $\ldots$ \\
\hline 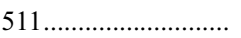 & 52,636 & 313 & 0.13 & 0.27 & $\ldots$ & $\ldots$ & $0.52(2.55)$ & $\ldots$ & $0.21(1.04)$ & $\ldots$ \\
\hline $512 \ldots \ldots \ldots \ldots \ldots \ldots \ldots \ldots \ldots \ldots \ldots \ldots \ldots \ldots \ldots \ldots . .$. & 51,992 & 302 & 0.05 & 0.23 & $0.98(0.24)$ & $\cdots$ & $0.00(18.95)$ & $-0.06(26.34)$ & $\ldots$ & $0.01(17.92)$ \\
\hline 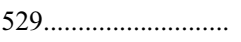 & 52,025 & 408 & 0.02 & 0.17 & $\ldots$ & $\ldots$ & $\ldots$ & $\ldots$ & $\ldots$ & $\ldots$ \\
\hline $550 \ldots \ldots \ldots \ldots \ldots \ldots \ldots \ldots \ldots \ldots \ldots \ldots \ldots \ldots$ & 51,959 & 624 & 0.17 & 0.20 & $\ldots$ & $\ldots$ & $\ldots$ & $\ldots$ & $\ldots$ & $\ldots$ \\
\hline 628.......................... & 52,083 & 262 & 0.03 & 0.12 & $\ldots$ & $\ldots$ & $\ldots$ & $\ldots$ & $\ldots$ & $\ldots$ \\
\hline $629 \ldots \ldots \ldots \ldots \ldots \ldots \ldots \ldots$ & 52,051 & 26 & 0.12 & 0.35 & $0.76(0.30)$ & $\ldots$ & $0.19(1.66)$ & $0.37(11.43)$ & $\ldots$ & $\ldots$ \\
\hline 637......................... & 52,174 & 334 & 0.09 & 0.18 & $1.12(0.50)$ & $\ldots$ & $0.00(5.20)$ & $0.22(2.16)$ & $\ldots$ & $\ldots$ \\
\hline 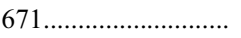 & 52,206 & 347 & 0.04 & 0.28 & $3.88(0.14)$ & $\ldots$ & $0.10(2.09)$ & $-0.33(2.66)$ & $\ldots$ & $-0.84(2.07)$ \\
\hline $765 \ldots \ldots \ldots \ldots \ldots \ldots \ldots \ldots$ & 52,254 & 179 & 0.04 & 0.21 & $\ldots$ & $\ldots$ & $\ldots$ & $\ldots$ & $\ldots$ & $\ldots$ \\
\hline $782 \ldots \ldots \ldots \ldots \ldots \ldots \ldots \ldots \ldots \ldots \ldots \ldots \ldots$ & 52,320 & 306 & 0.15 & 0.21 & $\ldots$ & $\ldots$ & $\ldots$ & $\ldots$ & $\ldots$ & $\ldots$ \\
\hline $790 \ldots \ldots \ldots \ldots \ldots \ldots \ldots \ldots$ & 52,441 & 527 & 0.03 & 0.14 & $\ldots$ & $\ldots$ & $-0.03(1.43)$ & $\ldots$ & $\ldots$ & $\ldots$ \\
\hline $792 \ldots \ldots \ldots \ldots \ldots \ldots \ldots \ldots$ & 52,353 & 432 & 0.14 & 0.20 & $1.88(0.45)$ & $\ldots$ & $0.15(2.90)$ & $0.07(1.81)$ & $\ldots$ & $0.08(2.16)$ \\
\hline $808 \ldots \ldots \ldots \ldots \ldots \ldots \ldots \ldots$ & 52,556 & 39 & 0.21 & 0.37 & $\ldots$ & $\ldots$ & $\ldots$ & $\ldots$ & $\ldots$ & $\ldots$ \\
\hline $845 \ldots \ldots \ldots \ldots \ldots \ldots \ldots \ldots$ & 52,381 & 270 & 0.07 & 0.32 & $\ldots$ & $\ldots$ & $\ldots$ & $\ldots$ & $\ldots$ & $\ldots$ \\
\hline $848 \ldots \ldots \ldots \ldots \ldots \ldots \ldots \ldots$ & 52,669 & 602 & 0.05 & 0.22 & $1.44(0.16)$ & $\ldots$ & $0.22(1.84)$ & $0.65(1.67)$ & $\ldots$ & $0.30(1.71)$ \\
\hline 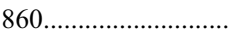 & 52,319 & 451 & 0.04 & 0.16 & $1.54(0.22)$ & $0.35(1.93)$ & $0.28(1.73)$ & $0.37(1.69)$ & $0.37(1.29)$ & $0.42(1.93)$ \\
\hline $864 \ldots \ldots \ldots \ldots \ldots \ldots \ldots \ldots$ & 52,320 & 91 & 0.04 & 0.26 & $1.89(0.25)$ & $\ldots$ & $-0.17(3.73)$ & $-0.20(3.50)$ & $\ldots$ & $-0.05(3.88)$ \\
\hline 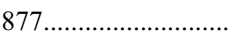 & 52,353 & 458 & 0.04 & 0.06 & $\ldots$ & $\ldots$ & $\ldots$ & $\ldots$ & $\ldots$ & $\ldots$ \\
\hline 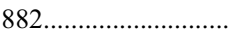 & 52,370 & 122 & 0.06 & 0.18 & $\ldots$ & $\ldots$ & $\ldots$ & $\ldots$ & $\ldots$ & $\ldots$ \\
\hline $883 \ldots \ldots \ldots \ldots \ldots \ldots \ldots \ldots$ & 52,430 & 366 & 0.05 & 0.13 & $2.78(0.55)$ & $\ldots$ & $-0.18(17.96)$ & $-0.09(2.66)$ & $\ldots$ & $\ldots$ \\
\hline 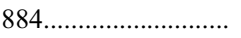 & 52,374 & 242 & 0.04 & 0.17 & $\ldots$ & $\ldots$ & $\ldots$ & $\ldots$ & $\ldots$ & $\ldots$ \\
\hline 889 & 52,663 & 408 & 0.06 & 0.16 & $\ldots$ & $\ldots$ & $\ldots$ & $\ldots$ & $\ldots$ & $\ldots$ \\
\hline 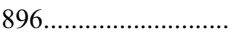 & 52,592 & 463 & 0.03 & 0.11 & $2.10(0.20)$ & $0.35(2.16)$ & $0.16(6.66)$ & $0.02(2.51)$ & $0.47(1.58)$ & $0.09(4.17)$ \\
\hline 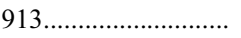 & 52,433 & 151 & 0.08 & 0.16 & $\ldots$ & $\ldots$ & $-0.10(1.79)$ & $\ldots$ & $\ldots$ & $\ldots$ \\
\hline 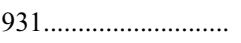 & 52,619 & 84 & 0.18 & 0.28 & $\ldots$ & $\ldots$ & $\ldots$ & $\ldots$ & $\ldots$ & $\ldots$ \\
\hline $958 \ldots \ldots \ldots \ldots \ldots \ldots \ldots \ldots \ldots$ & 52,410 & 194 & 0.15 & 0.31 & $4.08(0.77)$ & $\ldots$ & $-0.55(1.84)$ & $0.04(2.64)$ & $\ldots$ & $0.00(1.03)$ \\
\hline 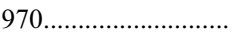 & 52,413 & 408 & 0.30 & 0.38 & $\ldots$ & $\ldots$ & $\ldots$ & $\ldots$ & $\ldots$ & $\ldots$ \\
\hline $980 \ldots \ldots \ldots \ldots \ldots \ldots \ldots \ldots$ & 52,431 & 300 & 0.13 & 0.29 & $0.99(0.28)$ & $\ldots$ & $0.00(1.73)$ & $-0.27(115.35)$ & $\ldots$ & $-0.28(11.09)$ \\
\hline 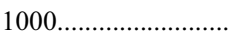 & 52,643 & 337 & 0.09 & 0.32 & $\ldots$ & $\cdots$ & $\ldots$ & $\ldots$ & $\cdots$ & $\ldots$ \\
\hline 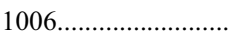 & 52,708 & 624 & 0.05 & 0.13 & $1.94(0.25)$ & $\cdots$ & $0.39(1.83)$ & $0.21(1.92)$ & $\ldots$ & $0.40(38.38)$ \\
\hline $1007 \ldots \ldots \ldots \ldots \ldots \ldots \ldots \ldots$ & 52,706 & 519 & 0.05 & 0.20 & $\ldots$ & $\ldots$ & $\ldots$ & $\ldots$ & $\ldots$ & $\ldots$ \\
\hline 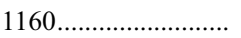 & 52,674 & 468 & 0.24 & 0.29 & $\ldots$ & $\ldots$ & $\ldots$ & $\ldots$ & $\ldots$ & $\ldots$ \\
\hline $1215 \ldots \ldots \ldots \ldots \ldots \ldots \ldots \ldots \ldots \ldots \ldots \ldots \ldots \ldots \ldots$ & 52,725 & 288 & 0.27 & 0.33 & $\ldots$ & $\ldots$ & $\ldots$ & $\ldots$ & $\ldots$ & $\ldots$ \\
\hline $1230 \ldots \ldots \ldots \ldots \ldots \ldots \ldots \ldots$ & 52,672 & 639 & 0.05 & 0.16 & $1.61(0.48)$ & $\ldots$ & $0.09(13.43)$ & $0.08(1.68)$ & $\ldots$ & $0.10(1.29)$ \\
\hline $1235 \ldots \ldots \ldots \ldots \ldots \ldots \ldots \ldots \ldots \ldots \ldots \ldots \ldots$ & 52,734 & 164 & 0.10 & 0.33 & $2.89(0.71)$ & $\ldots$ & $0.03(1.11)$ & $-0.09(2.04)$ & $\ldots$ & $-0.07(3.27)$ \\
\hline $1269 \ldots \ldots \ldots \ldots \ldots \ldots \ldots \ldots$ & 52,937 & 485 & 0.03 & 0.19 & $\ldots$ & $\ldots$ & $\ldots$ & $\ldots$ & $\ldots$ & $\ldots$ \\
\hline $1280 \ldots \ldots \ldots \ldots \ldots \ldots \ldots \ldots$ & 52,738 & 125 & 0.08 & 0.21 & $\ldots$ & $\ldots$ & $\ldots$ & $\ldots$ & $\ldots$ & $\ldots$ \\
\hline $1282 \ldots \ldots \ldots \ldots \ldots \ldots \ldots \ldots$ & 52,759 & 630 & 0.12 & 0.28 & $0.52(0.29)$ & $0.32(4.08)$ & $0.25(2.51)$ & $0.09(3.18)$ & $\ldots$ & $0.12(1.75)$ \\
\hline $1310 \ldots \ldots \ldots \ldots \ldots \ldots \ldots \ldots \ldots \ldots \ldots \ldots \ldots \ldots$ & 53,033 & 202 & 0.11 & 0.18 & $\ldots$ & $\ldots$ & $\ldots$ & $\ldots$ & $\ldots$ & $\ldots$ \\
\hline $1317 \ldots \ldots \ldots \ldots \ldots \ldots \ldots \ldots$ & 52,765 & 9 & 0.10 & 0.20 & $\ldots$ & $\ldots$ & $\ldots$ & $\ldots$ & $\ldots$ & $\ldots$ \\
\hline $1321 \ldots \ldots \ldots \ldots \ldots \ldots \ldots \ldots$ & 52,764 & 461 & 0.15 & 0.22 & $4.61(0.40)$ & $\ldots$ & $0.16(1.95)$ & $0.09(6.27)$ & $\ldots$ & $0.00(1.99)$ \\
\hline $1324 \ldots \ldots \ldots \ldots \ldots \ldots \ldots$ & 53,088 & 50 & 0.04 & 0.23 & $1.29(0.21)$ & $\ldots$ & $0.04(129.61)$ & $0.02(11.83)$ & $\ldots$ & $0.00(7.32)$ \\
\hline $1325 \ldots \ldots \ldots \ldots \ldots \ldots \ldots \ldots$ & 52,762 & 313 & 0.12 & 0.21 & $\ldots$ & $\ldots$ & $\ldots$ & $\ldots$ & $\ldots$ & $\ldots$ \\
\hline $1327 \ldots \ldots \ldots \ldots \ldots \ldots \ldots \ldots$ & 52,781 & 252 & 0.08 & 0.14 & $\ldots$ & $\ldots$ & $\ldots$ & $\ldots$ & $\ldots$ & $\ldots$ \\
\hline $1332 \ldots \ldots \ldots \ldots \ldots \ldots \ldots \ldots \ldots \ldots \ldots \ldots \ldots \ldots$ & 52,781 & 469 & 0.28 & 0.32 & $3.87(0.90)$ & $\cdots$ & $-0.99(3.02)$ & $-1.28(21.59)$ & $\ldots$ & $-0.99(1.44)$ \\
\hline $1363 \ldots \ldots \ldots \ldots \ldots \ldots \ldots \ldots$ & 53,053 & 104 & 0.18 & 0.27 & $2.02(0.44)$ & $\ldots$ & $0.25(11.95)$ & $0.21(1.32)$ & $\ldots$ & $\ldots$ \\
\hline
\end{tabular}


TABLE 5-Continued

\begin{tabular}{|c|c|c|c|c|c|c|c|c|c|c|}
\hline Plate & MJD & Fiber & $z_{\mathrm{fg}}$ & $z_{\mathrm{bg}}$ & $R$ & $\tau_{g r}$ & $\tau_{r i}$ & $\tau_{i z}$ & $\tau_{g r i}$ & $\tau_{\text {griz }}$ \\
\hline $1373 \ldots \ldots \ldots \ldots \ldots \ldots \ldots$ & 53,063 & 255 & 0.03 & 0.13 & $\ldots$ & $\ldots$ & $\ldots$ & $\ldots$ & $\ldots$ & $\ldots$ \\
\hline $1390 \ldots \ldots \ldots \ldots \ldots \ldots \ldots \ldots$ & 53,142 & 385 & 0.13 & 0.29 & $1.17(0.54)$ & $\ldots$ & $0.17(4.26)$ & $0.23(4.71)$ & $\ldots$ & $\ldots$ \\
\hline 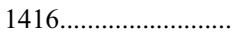 & 52,875 & 490 & 0.07 & 0.19 & $\ldots$ & $\ldots$ & $\ldots$ & $\ldots$ & $\ldots$ & $\ldots$ \\
\hline $1423 \ldots \ldots \ldots \ldots \ldots \ldots \ldots \ldots \ldots \ldots \ldots \ldots \ldots \ldots$ & 53,167 & 528 & 0.20 & 0.33 & $\ldots$ & $\ldots$ & $-0.31(1.17)$ & $\ldots$ & $\ldots$ & $\ldots$ \\
\hline $1429 \ldots \ldots \ldots \ldots \ldots \ldots \ldots \ldots$ & 52,990 & 336 & 0.07 & 0.15 & $1.27(0.15)$ & $0.00(4.62)$ & $0.48(2.55)$ & $-1.11(8.44)$ & $0.97(3.81)$ & $0.78(1.85)$ \\
\hline $1604 \ldots \ldots \ldots \ldots \ldots \ldots \ldots . .$. & 53,078 & 459 & 0.08 & 0.17 & $0.90(0.52)$ & $\ldots$ & $0.64(8.19)$ & $-0.04(4.06)$ & $\ldots$ & $0.82(1.06)$ \\
\hline 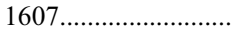 & 53,083 & 48 & 0.08 & 0.15 & $\ldots$ & $\ldots$ & $\ldots$ & $\ldots$ & $\ldots$ & $\ldots$ \\
\hline 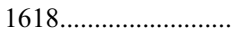 & 53,116 & 285 & 0.04 & 0.17 & $\ldots$ & $\ldots$ & $\ldots$ & $\ldots$ & $\ldots$ & $\ldots$ \\
\hline 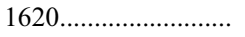 & 53,137 & 175 & 0.07 & 0.10 & $1.33(0.29)$ & $\ldots$ & $-0.11(3.35)$ & $-0.11(1.32)$ & $\ldots$ & $-0.07(11.45)$ \\
\hline 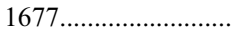 & 53,148 & 262 & 0.19 & 0.31 & $\ldots$ & $0.44(2.03)$ & $\ldots$ & $\ldots$ & $\ldots$ & $\ldots$ \\
\hline $1746 \ldots \ldots \ldots \ldots \ldots \ldots \ldots \ldots$ & 53,062 & 533 & 0.14 & 0.25 & $1.14(0.35)$ & $\ldots$ & $0.68(8.73)$ & $0.44(2.08)$ & $\cdots$ & $\cdots$ \\
\hline
\end{tabular}

Notes.- These values have not been corrected for inclination. Uncertainties are in parentheses.

We do not have meaningful extinction measurements close to the center of the foreground spiral, as there is not enough flux from the background galaxy. In a deeper image, there would be significant flux from the background elliptical nearer to the center of the spiral, enough to make a significant extinction measure there. The pairs did get selected, however, because both fell within the $3^{\prime \prime}$ of the SDSS spectroscopic aperture. Together with the resolution to distinguish between arm and disk, this is another argument for follow-up with HST, or possibly a large groundbased telescope.

\section{DISCUSSION AND CONCLUSIONS}

The occulting galaxy technique is a well-established one to measure disk extinction in the local universe. In combination with the wealth of data in the SDSS, we can construct a recent history of average disk extinction since $z \sim 0.3$. However, due to the spatial resolution, this disk opacity history is for the mix or arm and disk regions for all late-type disks. The redder optical bands have proven themselves to be optimal for opacity measurements in these pairs due to the brightness of the background early-type galaxy and less asymmetry in the foreground spiral.

From our analysis of 83 occulting pairs in the SDSS DR4 we can conclude the following:

1. Selection of occulting galaxy pairs from a uniform spectroscopic sample like the SDSS has a very high rate of success resulting in a substantial sample ( $(3)$.

2. Fits to occulting pairs can be automated, yet the optimal placement of an aperture to measure the disk opacity cannot $(\S 2$, Figs. 6 and 7).

3. The $i^{\prime}$ and $r^{\prime}$ filters in SDSS are optimal for measuring disk opacity in more distant galaxy pairs (Fig. 6).

4. The radial plots are similar to those found by White et al. (2000) for a mix of arm and disk values (Fig. 11).

5. There is not enough redshift range in the current sample with successful fits to distinguish an evolutionary trend in disk opacity (Fig. 11).

TABLE 6

Exponential Fit Values to Visual Aperture Optical Depths in the $r+i$

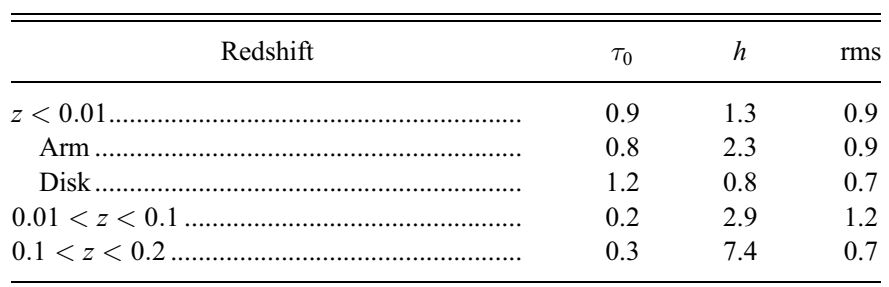

6. There is no relation between overall disk luminosity and local disk opacity, and only a weak relation between local surface brightness and disk opacity, probably because arms cannot be resolved at these distances by SDSS (Figs. 8 and 9).

7. Optical depth values in different filters agree with scatter due to the different asymmetry in galaxies in different filters, consistent with earlier findings (Fig. 10 ).

8. Stacking SDSS images does result in some $\mathrm{S} / \mathrm{N}$ improvements. In order to probe the inner parts of the spirals, deeper exposures are needed (Fig. 11).

9. The exponential profile of disk opacity for the higher redshift pairs is flatter than that of the nearby ones from Domingue et al. (2000). More points at lower galactic radius are needed to confirm (Fig. 11).

\section{FUTURE WORK}

These SDSS pairs constitute the ideal sample for study of spiral disks opacity in the local universe $(z<0.4)$. For these and more distant pairs, the photometric stability and high resolution of HST will be needed to (1) resolve the Hubble subtype and (2) distinguish

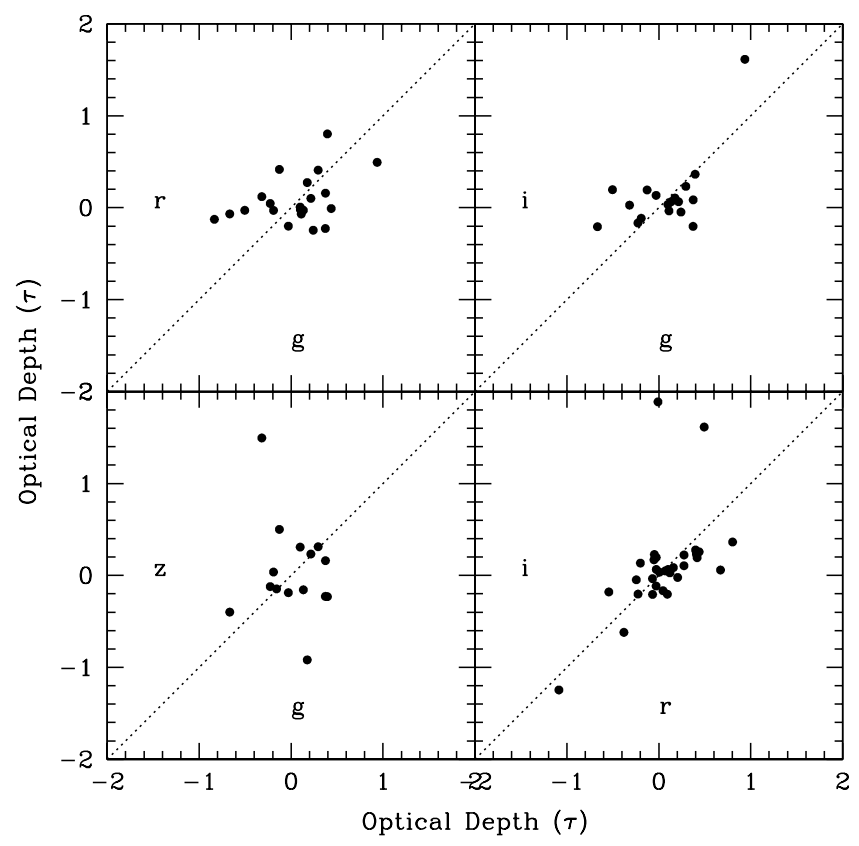

FIG. 10.- Relation between opacity measurements in the visual aperture for four different SDSS filters $\left(g^{\prime}, r^{\prime}, i^{\prime}, z^{\prime}\right)$. Only the nonzero opacities are shown. The values agree for the most part for the visual aperture, as a Galactic extinction law is expected to be resolved only when the spatial scale is less than $100 \mathrm{pc}$. 


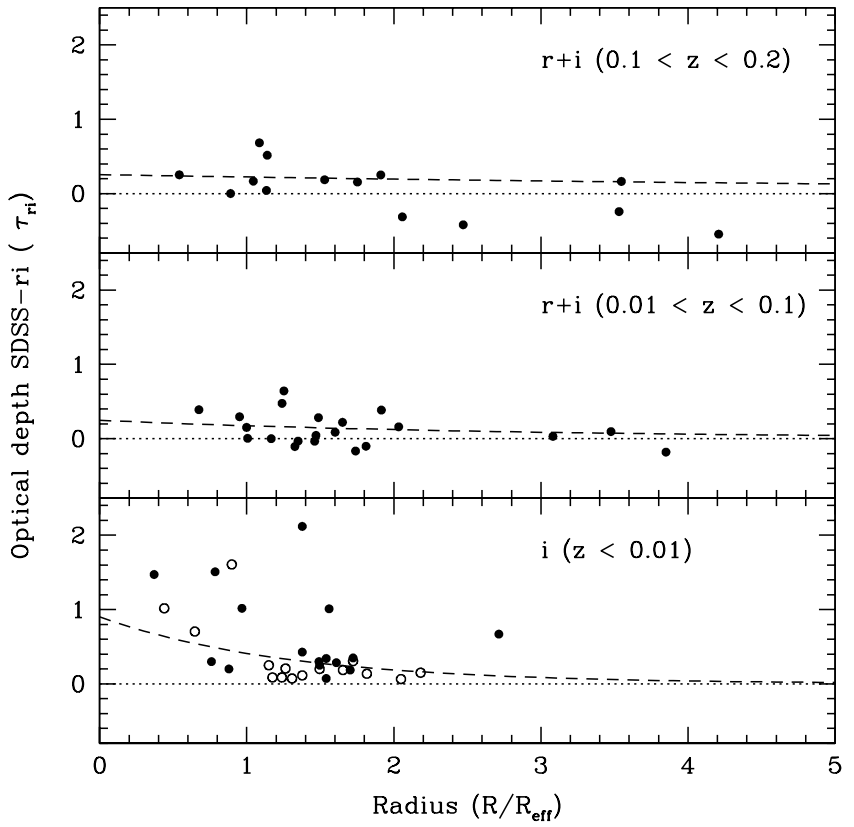

FIG. 11.-Radial profile from Fig. 14 (bottom) in Domingue et al. (2000) and the optical depths profile from the $r+i$ stacked image. The $R_{25}$ from Domingue et al. (2000) was converted to effective radius with $R_{25}=1.43 R_{\text {eff }}$. Open circles in the bottom panel represent disk regions, and filled circles represent spiral arms in the case of the Domingue et al. (2000) values. Exponential disk fits to the points are shown by dashed lines. between arm and disk sections and allow for optical depth values closer to the center of the spiral. The SDSS measurements can, however, serve as a reference for higher redshift pairs images with $H S T$ for which Hubble type and spiral arm identification also remain impossible.

The authors would like to thank Roelof de Jong, Ron Allen, and Harry Ferguson for useful discussions. We thank Lorraine and Steve Mencinsky for assistance in examining each candidate pair for suitable geometry. Funding for the Sloan Digital Sky Survey (SDSS) has been provided by the Alfred P. Sloan Foundation, the Participating Institutions, the National Aeronautics and Space Administration, the National Science Foundation, the US Department of Energy, the Japanese Monbukagakusho, and the Max Planck Society. The SDSS Web site is at http://www.sdss.org/.

The SDSS is managed by the Astrophysical Research Consortium for the Participating Institutions. The Participating Institutions are the University of Chicago, Fermilab, the Institute for Advanced Study, the Japan Participation Group, The Johns Hopkins University, Los Alamos National Laboratory, the Max Planck Institute for Astronomy, the Max Planck Institute for Astrophysics, New Mexico State University, the University of Pittsburgh, Princeton University, the US Naval Observatory, and the University of Washington. W. C. K. is grateful for support from a Dean's Leadership Board Faculty Fellowship.
Andredakis, Y. C., \& van der Kruit, P. C. 1992, A\&A, 265, 396 Berlind, A. A., et al. 1997, AJ, 114, 107

Bertin, E., \& Arnouts, S. 1996, A\&AS, 117, 393

Bolton, A. 2005, HST Proposal 6907

Bolton, A. S., et al. 2004, AJ, 127, 1860 2006, ApJ, 638, 703

Calzetti, D., \& Heckman, T. M. 1999, ApJ, 519, 27

Domingue, D. L., et al. 1999, AJ, 118, 1542 . 2000, ApJ, 545, 171

Elíasdóttir, Á., et al. 2006, ApJS, 166, 443

Elmegreen, D. M., et al. 2001, AJ, 121, 182

Falco, E. E., et al. 1999, ApJ, 523, 617

Fukugita, M., et al. 1996, AJ, 111, 1748

Gavazzi, R., et al. 2007, ApJ, 667, 176

Gunn, J. E., et al. 1998, AJ, 116, 3040

Holwerda, B. W. 2005, preprint (astro-ph/0512139)

Holwerda, B. W., et al. 2005a, AJ, 129, 1396 2005b, A\&A, 444, 109

Hopkins, P. F., et al. 2004, AJ, 128, 1112

Jha, S., et al. 2007, ApJ, 659, 122

Keel, W. C., \& White, R. E. 2001a, AJ, 121, 1442

\section{REFERENCES}

Keel, W. C., \& White, R. E. 2001b, AJ, 122, 1369

Knop, R. A., et al. 2003, ApJ, 598, 102

Koopmans, L. 2004, HST Proposal 6492

Koopmans, L. V. E., et al. 2006, ApJ, 649, 599

Masters, K. L., et al. 2003, AJ, 126, 158

Motta, V., et al. 2002, ApJ, 574, 719

Nadeau, D., et al. 1991, ApJ, 376, 430

Nilsson, K. K., et al. 2007, A\&A, 471, 71

Rowan-Robinson, M. 2003, MNRAS, 344, 13

Rowan-Robinson, M., et al. 2005, AJ, 129, 1183

Sajina, A., et al. 2006, MNRAS, 369, 939

Smith, J. A., et al. 2002, AJ, 123, 2121

Treu, T., et al. 2006, ApJ, 640, 662

Tully, R. B., et al. 1998, AJ, 115, 2264

Vijh, U. P., et al. 2003, ApJ, 587, 533

Warren, S. J., et al. 1996, MNRAS, 278, 139

White, R. E., \& Keel, W. C. 1992, Nature, 359, 129

White, R. E., et al. 2000, ApJ, 542, 761

Yan, H., et al. 2005, ApJ, 634, 109

York, D. G., et al. 2000, AJ, 120, 1579 